

\title{
A MULTIDOMAIN SPECTRAL COLLOCATION METHOD FOR THE STOKES PROBLEM
}

\author{
G. Sacchi Landriani \\ Istituto di Analisi Numerica del CNR \\ 41833166 \\ Corso Carlo Alberto 5 \\ 27100 Pavia, Italy \\ H. Vandeven ${ }^{1}$ \\ Laboratoire Analyse Numérique \\ Université P. et M. Curie \\ tour 45-55 4 Place Jussieu \\ 75230 Paris Cedex 05, France
}

\begin{abstract}
We propose a multidomain spectral collocation scheme for the approximation of the two-dimensional Stokes problem. We show that the discrete velocity vector field is exactly divergence-free and we prove error estimates both for the velocity and the pressure.
\end{abstract}

\footnotetext{
${ }^{1}$ This research was supported by the National Aeronautics and Space Administration under NASA Contract No. NAS1-18107 while the second author was in residence at the Institute for Computer Applications in Science and Engineering (ICASE), NASA Langley Research Center, Hampton, VA 23665.
} 
.

$\equiv$

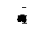

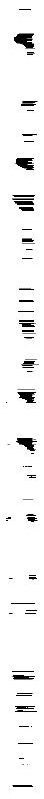

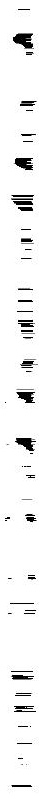

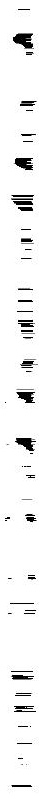

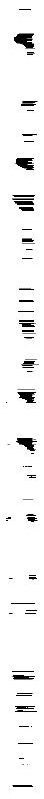

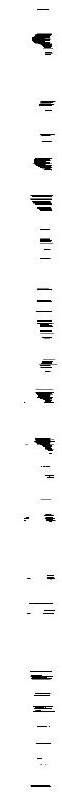

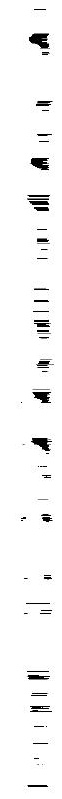

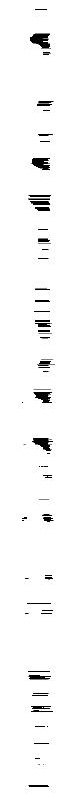

$\equiv$
$\equiv$

$\bar{\equiv}$
$\overline{\overline{\underline{E}}}$

$\overline{-}$

$=$
$=$

$\equiv$
$\equiv$
$\bar{E}$ 


\section{Introduction.}

Domain decomposition methods for the approximation of partial differential equations are based on the partition of the physical domain into subdomains. These techniques allow one to decompose the problem into a collection of problems of smaller size, defined on the subdomains. By using proper interface conditions the overall efficiency of the algorithm is then improved.

If spectral schemes are considered domain decomposition methods may also be used to handle problems defined on plurirectangular domains. Actually, spectral methods are naturally defined only on rectangular domains (see e.g. [13]). If a problem on a plurirectangle is considered then it is possible to decompose such a domain in rectangles and employ spectral schemes on these subdomains. For the analysis of spectral multidomain methods for the Helmholtz equation we refer to $[7],\{9],\{11],[12],,[16],\{18],[19]$. General considerations on domain decomposition in the framework of spectral methods can be found in [8, Ch.13].

In this paper we consider the Stokes problem in a plurirectangular domain with homogeneous Dirichlet boundary conditions. We analyze a scheme obtained from a generalization of the spectral collocation method introduced in [6] and [15] for the case of a monodomain approximation. An interesting feature of the numerical scheme is that, for any value of the discretization parameter, the approximated velocity field is exactly divergence-free. This is an important property in itself because it ensures that, even if a small number of degrees of freedom is used, the continuity equation is solved exactly. Furthmore, if the Stokes solver is used at each step of a time discretization of the full Navier-Stokes equation, then this property makes the timealgorithm more stable.

Our main interest lies in the analysis of the discrete problem and in the proof of error estimates both for the velocity and the pressure. The number of subdomains is considered fixed and convergence is achieved when the degree of the solution (which is a polynomial in each subdomain) tends to infinity. Iterative methods for solving the discrete problem are under investigation and some related numerical experiments are being conducted.

A multidomain scheme for the approximation of the Stokes problem can be found in [16] where the "Spectral Element" method is presented. The main difference between that method and the one we analyze here lies in the choice of the discrete pressure space and in the quadrature formulae which are employed. Domain decomposition for solving the Stokes problem in the framework of the Finite Element method is treated e.g. in [17] and [14].

The paper is organized as follows. In section one we introduce the continuous Stokes problem. A first version of the discrete problem is then formulated in a variational form and interpreted in a collocation one. Throughout the section we assume, for the sake of simplicity, that the physical domain is a rectangle partitioned in aligned subrectangles. We show that the discrete velocity is exactly divergence-free and we state an inf-sup condition which garantees compatibility between the velocity space and the pressure space (see [2]). In section two we give a new formulation of the discrete problem, which is more appropiate for the computation of the pressure. 
Using results from the previous section, we prove convergence results both for the velocity and the pressure. In section three we consider the case of a plurirectangular domain and we extend the results of the previous sections. Finally an appendix is devoted to the proof of the inf-sup condition stated in section one.

\section{Notation.}

In the following $M$ is a fixed positive integer and $\Omega$ is a plurirectangle partitioned in rectangles $\Omega_{i}, i=1, \ldots, M$. Any quantity defined in $\Omega_{i}$ is identified by a superscript index $i$. For instance if $v$ is a function defined on $\Omega$ then the restriction of $v$ to $\Omega_{i}$ is denoted by $v^{i}$. We shall use the classical Sobolev spaces $H^{*}(\Omega), H^{*}\left(\Omega_{i}\right)$, $i=1, \ldots, M, s \in \mathrm{R}$ (see e.g. [1]). The norms of these spaces will be denoted by $\|\cdot\|_{\odot}$ and $\|\cdot\|_{\odot, i}$ respectively.

For any positive integer $N$ we denote by $P_{N}\left(\bar{\Omega}_{i}\right)$ the space of the restrictions to $\bar{\Omega}_{i}$ of the polynomials of degree less or equal to $N$ with respect to each variable. For any integer $i, 1 \leq i \leq M$, let $\Xi_{N}^{i}$ be the set of the $(N+1)^{2}$ nodes related to the Legendre Gauss Lobatto quadrature formula in $\bar{\Omega}_{i}$. The points of $\Xi_{N}^{i}$ are denoted by $\left(x_{k}^{i}, y_{l}^{i}\right), 1 \leq k, l \leq N$ and the related quadrature formula is

$$
\int_{\Omega_{i}} v(x, y) d x d y \simeq \sum_{k=0}^{N} \sum_{l=0}^{N} v\left(x_{k}^{i}, y_{l}^{i}\right) \omega_{k}^{i} \omega_{l}^{i},
$$

where $\omega_{k}^{i}$ and $\omega_{l}^{i}$ are positive weights (see e.g. [10]). We recall that if $v \in P_{2 N-1}\left(\bar{\Omega}_{i}\right)$ then the quadrature formula $(0.1)$ is exact. For any $v, w \in C^{0}\left(\bar{\Omega}_{i}\right)$ we set

$$
(v, w)_{N, i}=\sum_{k=0}^{N} \sum_{l=0}^{N} v\left(x_{k}^{i}, y_{l}^{i}\right) \omega_{k}^{i} \omega_{l}^{i}, \quad i=1, \ldots, M .
$$

This bilinear form is an inner product on $P_{N}\left(\bar{\Omega}_{i}\right)$, moreover we have (see [8]):

$$
C_{1}\|v\|_{0, i} \leq\|v\|_{N, i} \leq C_{2}\|v\|_{0, i} \quad \forall v \in P_{N}\left(\bar{\Omega}_{i}\right), \quad i=1, \ldots, M,
$$

where $C_{1}$ and $C_{2}$ are positive comstants independent of $N$ and $\|\cdot\|_{N, i}$ is the norm associated to $(0.2)$. For any $v, w \in C^{0}(\bar{\Omega})$ we define

$$
((v, w))_{N}=\sum_{i=1}^{M}\left(v^{i}, w^{i}\right)_{N, i}
$$

This bilinear form is an inner product on $\prod_{i=1}^{M} P_{N}\left(\bar{\Omega}_{i}\right)$. Denoting by $\|\cdot\|_{N}$ the associated norm, we have

$$
C_{1}\|v\|_{0} \leq\|v\|_{N} \leq C_{2}\|v\|_{0} \quad \forall v \in \prod_{i=1}^{M} P_{N}\left(\bar{\Omega}_{i}\right),
$$


where $C_{1}$ and $C_{2}$ are positive constants independent of $N, M$ and of the measure of each subdomain. If $\mathbf{v}$ and $\mathrm{w}$ are vector valued functions we keep the same notation for the inner product and the norm induced by $(0.2)$ and $(0.4)$. Note that the form $((\cdot, \cdot))_{N}$ is an approximation of the $L^{2}$-inner product denoted by $(\cdot, \cdot)$.

Given an integer $i, 1 \leq i \leq M$, and a point $\mathrm{P}=\left(x_{k}^{i}, y_{l}^{i}\right) \in \Xi_{N}^{i}$ the characteristic polynomial associated to $\mathrm{P}$ is the element of $P_{N}\left(\bar{\Omega}_{i}\right)$ such that

$$
\chi_{\mathrm{P}}^{i}= \begin{cases}\frac{1}{\omega_{k}^{i} \omega_{l}^{i}} & \text { in } \mathrm{P} \\ 0 & \text { in the other points of } \Xi_{N}^{i} .\end{cases}
$$

Finally if $\bar{\Omega}_{i}$ is a rectangle given by $\left[x_{i-1}, x_{i}\right] \times\left[y_{i-1}, y_{i}\right]$ we define, for any $n \in N$ :

$$
\begin{aligned}
& L_{n}^{i}(x)=L_{n}\left(\frac{2 x-x_{i-1}-x_{i}}{x_{i}-x_{i-1}}\right) \\
& L_{n}^{i}(y)=L_{n}\left(\frac{2 y-y_{j-1}-y_{i}}{y_{i}-y_{i-1}}\right)
\end{aligned}
$$

where $L_{n}(\xi)$ is the Legendre polynomial of degree $n$ defined in $[-1,1]$, (see e.g. [10]). We also let $C$ denote a positive constant. The value of $C$ shall always be independent of the discretization parameter $N$. 


\section{The continuous and the discrete problems.}

In this section we consider the case of a rectangle $\Omega$ partitioned in aligned rectangle $\Omega_{i}, i=1, \ldots, M$, as in the figure 1.1. The interfaces between subdomains are denoted by $\Gamma_{i}, i=1, \ldots, M-1$.

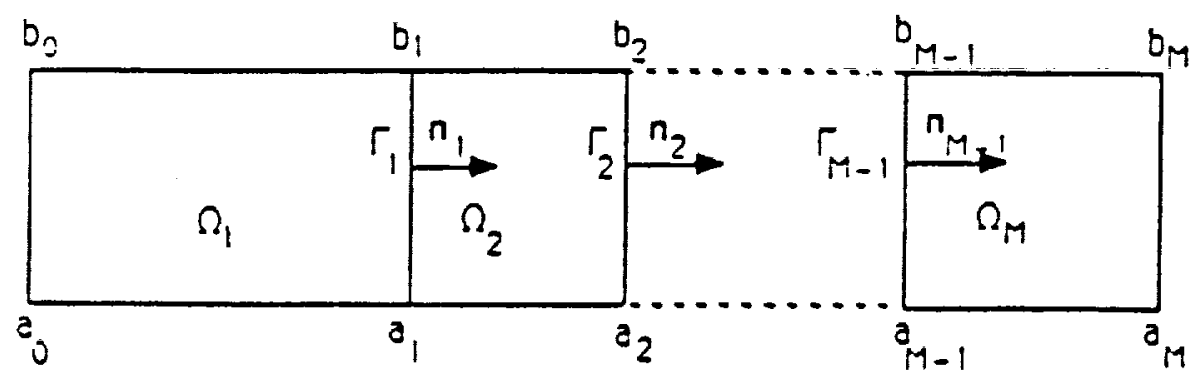

figure.1.1 Decomposition with aligned subdomains.

Let us consider the Stokes equations:

$$
\begin{gathered}
-\Delta \mathbf{u}+\nabla p=\mathbf{f} \quad \text { in } \Omega \\
\nabla \cdot \mathbf{u}=0 \quad \text { in } \Omega \\
\mathbf{u}=0 \quad \text { on } \partial \Omega .
\end{gathered}
$$

As usual $u$ and $p$ denote the velocity and the pressure of an incompressible fluid, the kinematic viscosity has been normalized to one and $f$ represents a source term. Supposing $f \in\left(L^{2}(\Omega)\right)^{2}$ it is well known that problem (1.1) has a unique solution in $\left(H^{2}(\Omega)\right)^{2} \times H^{1}(\Omega) / \mathrm{R}$ and that its variational formulation is the following (see e.g. [21]).

Find $\mathbf{u} \in\left(H_{0}^{1}(\Omega)\right)^{2}$ and $p \in L^{2}(\Omega) / \mathrm{R}$ such that

$$
\begin{gathered}
(\nabla \mathrm{u}, \nabla \mathrm{v})-(p, \nabla \cdot \mathrm{v})=(\mathrm{f}, \mathrm{v}) \quad \forall \mathrm{v} \in\left(H_{0}^{1}(\Omega)\right)^{2} \\
(\nabla \cdot \mathrm{u}, q)=0 \quad \forall q \in L^{2}(\Omega) / \mathrm{R} .
\end{gathered}
$$

We now define a multidomain spectral approximation of this problem. The velocity space $\left(H_{0}^{1}(\Omega)\right)^{2}$ is approximated by its finite dimensional subspace $V_{N}$ defined as follows:

$$
V_{N}=\left\{\mathrm{v} \in\left(C^{0}(\bar{\Omega})\right)^{2}: \mathrm{v}^{i} \in\left(P_{N}\left(\bar{\Omega}_{i}\right)\right)^{2}, \mathrm{v}^{i}=0 \text { on } \partial \Omega \cap \partial \Omega_{i}, \quad i=1, \ldots, M\right\}
$$

Denoting by $M_{N}$ the discrete pressure space (which is defined below) and recalling the definition (0.4), we consider the following approximation of problem (1.2). 
Find $\mathbf{u}_{N} \in V_{N}$ and $p_{N} \in M_{N}$ such that

$$
\begin{gathered}
\left(\left(\nabla \mathbf{u}_{N}, \nabla \mathbf{v}_{N}\right)\right)_{N}-\left(\left(p_{N}, \nabla \cdot \mathbf{v}_{N}\right)\right)_{N}=\left(\left(\mathbf{f}, \mathbf{v}_{N}\right)\right)_{N} \quad \forall \mathbf{v}_{N} \in V_{N} \\
\left(\left(\nabla \cdot \mathbf{u}_{N}, q_{N}\right)\right)_{N}=0 \quad \forall q_{N} \in M_{N} .
\end{gathered}
$$

Here we have supposed that $f \in\left(C^{0}(\bar{\Omega})\right)^{2}$. We require that $M_{N}$ is a subspace of $\prod_{i=1}^{M} P_{N}\left(\bar{\Omega}_{i}\right)$ such that problem (1.4) is well posed. Clearly $M_{N}$ must not contain any "spurious mode" of the pressure, i.e. any nonvanishing element of the space

$$
Z_{N, M}=\left\{q \in \prod_{i=1}^{M} P_{N}\left(\bar{\Omega}_{i}\right):((q, \nabla \cdot v))_{N}=0 \quad \forall v \in V_{N}\right\} .
$$

In the following ?pr position, which is proved in the appendix, we precise which are the elements of $Z_{N, M}$. Let us first remark that, due to the geometry of $\Omega$, the polynomials $L_{n}^{i}(y)$, defined in (0.6), do not depend on $i$; hence the index $i$ will be omitted.

Proposition 1.1. The space $Z_{N, M}$ is a linear subspace of $\prod_{i=1}^{M} P_{N}\left(\bar{\Omega}_{i}\right)$ whose dimension is $4 M+4$. A basis of $Z_{N, M}$ is given by the following elements.

$$
\begin{gathered}
\left(\chi_{a_{0}}^{1}, 0, \ldots, 0\right), \quad\left(\chi_{b_{0}}^{1}, 0, \ldots, 0\right) \\
\left(0, \ldots, 0, \chi_{a_{M}}^{M}\right), \quad\left(0, \ldots, 0, \chi_{b_{M}}^{M}\right) \\
\left(0, \ldots, 0, \chi_{a_{i}}^{i},-\chi_{a_{i}}^{i+1}, 0, \ldots, 0\right), \quad\left(0, \ldots, 0, \chi_{b_{i}}^{i},-\chi_{b_{i}}^{i+1}, 0, \ldots, 0\right) \text { for } i=1, \ldots, M-1 \\
(1,1, \ldots, 1),\left(L_{N}(y), L_{N}(y), \ldots, L_{N}(y)\right) \\
\left(0, \ldots, 0, L_{N}^{i}(x), 0, \ldots, 0\right), \quad\left(0, \ldots, 0, L_{N}^{i}(x) L_{N}(y), 0, \ldots, 0\right) \text { for } i=1, \ldots, M .
\end{gathered}
$$

We shall define the space $M_{N}$ so that $M_{N} \oplus Z_{N, M}=\prod_{i=1}^{M} P_{N}\left(\bar{\Omega}_{i}\right)$. A possible choice is

$$
M_{N}=\left\{q \in \prod_{i=1}^{M} P_{N}\left(\bar{\Omega}_{i}\right):((q, r))_{N}=0 \quad \forall r \in Z_{N, M}\right\} .
$$

Using (0.2)(0.4) and (1.6)-(1.10) it is readly seen that $M_{N}$ can be written as follows:

$$
\begin{aligned}
M_{N}=\{ & q \in \prod_{i=1}^{M} P_{N}\left(\bar{\Omega}_{i}\right): q^{1}\left(a_{0}\right)=q^{1}\left(b_{0}\right)=q^{M}\left(a_{M}\right)=q^{M}\left(b_{M}\right)=0, \\
& q^{i}\left(a_{i}\right)=q^{i+1}\left(a_{i}\right), q^{i}\left(b_{i}\right)=q^{i+1}\left(b_{i}\right), i=1, \ldots, M-1, \\
& \sum_{i=1}^{M}\left(q^{i}, 1\right)_{N, i}=0, \sum_{i=1}^{M}\left(q^{i}, L_{N}(y)\right)_{N, i}=0, \\
& \left.\left(q^{i}, L_{N}^{i}(x)\right)_{N, i}=0,\left(q^{i}, L_{N}^{i}(x) L_{N}(y)\right)_{N, i}=0, i=1, \ldots, M\right\} .
\end{aligned}
$$


Theorem 1.1. Problem (1.4) has a unique solution.

Proof. If $\mathrm{f}=0$ then choosing $\mathrm{v}_{N}=\mathrm{u}_{N}$ and $q_{N}=p_{N}$ in (1.4) we obtain $\left(\left(\nabla \mathbf{u}_{N}, \nabla \mathbf{u}_{N}\right)\right)_{N}=0$. From (1.3) and the Poincaré inequality this gives $\mathbf{u}_{N}=0$. From the proposition 1.1 we also deduce $p_{N}=0$ hence the theorem is proved.

For the couple of spaces $\left(V_{N}, M_{N}\right)$ the following "inf-sup" condition holds (see the appendix for the proof).

Theorem 1.2. There exists a positive constant $C$ independent of $N$ such that

$$
\inf _{q_{N} \in M_{N}} \sup _{\mathbf{v}_{N} \in V_{N}} \frac{\left(\left(q_{N}, \nabla \cdot \mathbf{v}_{N}\right)\right)_{N}}{\left\|q_{N}\right\|_{N}\left\|\nabla \mathbf{v}_{M}\right\|_{N}} \geq \frac{C}{N}
$$

Remark 1.2. Note that the right hand side of (1.13) depends on the discretization parameter $N$. This estimate is optim al, actually an upper bound for the left hand side of (1.13) is $C l / N$, where $C l$ is a positive constant independent of $N$. For a detailed discussion on this subject we refer to [20].

Proposition 1.2. If $\mathrm{v}_{N} \in V_{N}$ then $\nabla \cdot \mathrm{v}_{N} \in M_{N}$.

Proof. Since $\mathrm{v}_{N}=0$ on $\partial \Omega$ we have $\nabla \cdot \mathrm{v}=0$ at $a_{0}, b_{0}, a_{M}$, and $b_{M}$. Moreover $\mathrm{v}^{i}=\mathrm{v}^{i+1}$ on $\Gamma_{i}$ for $i=1, \ldots, M-1$, hence $\nabla \cdot v^{i}\left(a_{i}\right)=\nabla \cdot v^{i+1}\left(a_{i+1}\right)$ and $\nabla \cdot v^{i}\left(b_{i}\right)=$ $\nabla \cdot v^{i+1}\left(b_{i}\right)$, for $i=1, \ldots, M-1$. Recalling $(0.2)$ and the fact that the Legendre Gauss Lobatto formula is exact for polynomials of degree $2 N-1$, we have, from the divergence theorem

$$
\sum_{i=1}^{M}\left(\nabla \cdot v^{i}, 1\right)_{N, i}=0
$$

moreover

$$
\begin{aligned}
& \sum_{i=1}^{M}\left(\nabla \cdot v^{i}, L_{N}(y)\right)_{N, i}=\sum_{i=1}^{M}\left(v_{1, x}^{i}, L_{N}(y)\right)_{N, i}= \\
& =\sum_{i=1}^{M} \sum_{l=0}^{N} L_{N}\left(y_{l}\right) \int_{a_{i-1}}^{a_{i}} v_{1, x}^{i}\left(x, y_{l}\right) d x \omega_{l}^{i}=0 .
\end{aligned}
$$

Using the same arguments we also deduce

$$
\left(\nabla \cdot \mathbf{v}^{i}, L_{N}^{i}(x)\right)_{N, i}=\left(v_{2, y}^{i}, L_{N}^{i}(x)\right)_{N, i}=\sum_{k=0}^{N} L_{N}^{i}\left(x_{k}\right) \int_{a_{i}}^{b_{i}} v_{2, y}^{i}\left(x_{k}, y\right) d y \omega_{k}^{i}=0 .
$$

Finally, since $\mathbf{v}^{i} \in\left(P_{N}\left(\bar{\Omega}_{i}\right)\right)^{2}$ we have $\left(\nabla \cdot v^{i}, L_{N}^{i}(x) L_{N}(y)\right)_{i}=0$. It is readly seen that this implies $\left(\nabla \cdot v^{i}, L_{N}^{i}(x) L_{N}(y)\right)_{N, i}=0$, for $i=1, \ldots, M$.

The following is a direct consequence of (1.4) and Proposition 1.2. 
Theorem 1.3. If $\mathrm{u}_{N}$ is the solution of problem (1.4) then $\nabla \cdot \mathrm{u}_{N}^{i}=0$ in $\bar{\Omega}_{i}$ for $i=1, \ldots, M$.

Remark 1.2. Using the technique introduced in [6, Corollary 5.1], for the monodomain case, one can prove that $M_{N}$ is exactly the image of $V_{N}$ through the divergence operator.

We now give a collocation form of problem (1.4). Let us recall that $\Xi_{N}^{i} \cap \Omega_{i}$, for $1 \leq i \leq M$ is the set of the collocation points internal to $\Omega_{i}$, and $\Xi_{N}^{i} \cap \Gamma_{i}, 1 \leq$ $i \leq M-1$ is the set of the points which lies on the interfaces $\Gamma_{i}$ (not including the endpoints $a_{i}$ and $b_{i}$ ). Let now $\xi_{k}^{i}, k=1, \ldots, 4$, be four points of $\Xi_{N}^{1} \cap \Omega_{1}$ and let $\xi_{k}^{i}, k=1,2$ be two points of $\Xi_{N}^{i} \cap \Omega_{i}$, for $i=2, \ldots, M$. We suppose that

$$
\operatorname{det}\left\{\varphi_{l}^{1}\left(\xi_{k}^{1}\right)\right\} \neq 0
$$

where $\varphi_{l}^{1}, l=1, \ldots, 4$ are the four polynomials $1, L_{N}^{1}(x), L_{N}(y), L_{N}^{1}(x) L_{N}(y)$. Moreover denoting by $\psi_{l}^{i}, l=1,2, i=2, \ldots, M$ the polynomials $L_{N}^{i}(x)$ and $L_{N}^{i}(x) L_{N}(y)$, we suppose that

$$
\operatorname{det}\left\{\psi_{l}^{i}\left(\xi_{k}^{i}\right)\right\} \neq 0 \text { for } i=2, \ldots, M .
$$

The hypothesis (1.14) and (1.15) are for instance verified if for any $i=1, \ldots, M$ the points $\xi_{l}, l=1,2$ (or $l=1, \ldots, 4$ if $i=1$ ), are not aligned.

We can now state the following.

Proposition 1.9. Problem (1.4) is equivalent to find $\mathrm{u}_{N} \in V_{N}$ and $p_{N} \in M_{N}$ such that

$$
\begin{aligned}
& -\Delta \mathbf{u}_{N}^{i}(\xi)+\nabla p_{N}^{i}(\xi)=f^{i}(\xi) \quad \forall \xi \in \Xi_{N}^{i} \cap \Omega_{i} \text { for } i=1, \ldots, M, \\
& \nabla \cdot \mathbf{u}_{N}^{1}(\xi)=0 \quad \forall \xi \in \Xi_{N}^{1} \text { exept } \xi \in\left\{a_{0}, b_{0}, a_{1}, b_{1}, \xi_{k}^{1}, k=1, \ldots, 4\right\} \\
& \nabla \cdot \mathbf{u}_{N}^{i}(\xi)=0 \quad \forall \xi \in \Xi_{N}^{i} \text { exept } \xi \in\left\{a_{i}, b_{i}, \xi_{k}^{i}, k=1,2\right\} \text { for } i=2, \ldots, M, \\
& \left(\frac{\partial \mathbf{u}_{N}^{i}}{\partial \mathbf{n}^{i}}-p_{N}^{i} \mathbf{n}^{i}\right)(\xi)-\left(\frac{\partial \mathbf{u}_{N}^{i+1}}{\partial \mathbf{n}^{i}}-p_{N}^{i+1} \mathbf{n}^{i}\right)(\xi)= \\
& =-\sum_{j=i}^{i+1}\left\{\left(-\Delta \mathbf{u}_{N}^{j}+\nabla p_{N}^{j}-f^{j}\right) \omega_{N}^{j}\right\}(\xi) \quad \forall \xi \in \Xi_{N}^{i} \cap \Gamma_{i} i=1, \ldots, M-1
\end{aligned}
$$

We omit the proof of this proposition because it is a direct generalization of the proof given in [6, Prop.5.1] for the monodomain case. Let us however precise some remarks about (1.16)-(1.19). In (1.16) we impose the momentum equation at the internal collocation nodes of each subdomain. These equations are obtained by (1.4) by choosing $\mathbf{v}_{N}$ as the characteristic function of the internal nodes of $\Omega_{i}, i=1, \ldots, M$ and by using (0.2). In (1.17) and (1.18) the continuity equation is imposed in all the 
collocation points exept a set of $4 M+4$ points. In order to have the same number of unknowns and equations it is necessary to eliminate such points (we recall that $M_{N}$ is a subspace of codimension $4 M+4$ of $\left.\prod_{i=1}^{M} P_{N}\left(\bar{\Omega}_{i}\right)\right)$. We enphasize that the solution of (1.16)-(1.19) do not depend on the choice of the points $\xi_{k}^{1}, k=1, \ldots, 4$ and $\xi_{k}^{i}, k=1,2, i=2, \ldots, M$, provided the hypothesis (1.14) and (1.15) hold. Concerning (1.19), note that on the right hand side we have the residual of the momentum equation multiplied by the weight $\omega_{N}^{j}$ (which behaves like $N^{-2}$ ). Hence (1.19) asymptotically ensures that the stress tensor is continuous at the interfaces $\Gamma_{i}, i=1, \ldots, M-1$. This property is clearly verified by the solution of the continuous problem (1.1).

\section{Error estimates and a new pressure space.}

In this section we first prove an error estimate for the velocity field.

Let $\mathrm{w} \in\left(H_{0}^{1}(\Omega)\right)^{2}$, (where $\Omega$ is given in figure 1.1), be such that $\nabla \cdot \mathrm{w}=0$ in $\Omega$. We denote by $\Pi_{N}^{1, d i v} w$ the orthogonal projection of $w$, for the inner product of $\left(H_{0}^{1}(\Omega)\right)^{2}$, on the space of the divergence-free functions which belong to $V_{N}$. The following approximation result holds.

Theorem 2.1. Let $\mathrm{w} \in\left(H_{0}^{1}(\Omega)\right)^{2}$ be a divergence-free function and suppose that $\mathbf{w}^{i} \in\left(H^{\sigma_{i}}\left(\Omega_{i}\right)\right)^{2}$, with $\sigma_{i} \geq 1$, for $i=1, \ldots, M$. There exists a positive constant $C$ independent of both $\mathrm{w}$ and $N$ such that

$$
\left\|\mathrm{w}-\Pi_{N}^{1, d i v} \mathrm{w}\right\|_{1} \leq C \sum_{i=1}^{M} N^{1-\sigma_{i}}\left\|\mathrm{w}^{i}\right\|_{\sigma_{i}, i} .
$$

Proof. Since $\mathrm{w} \in\left(H_{0}^{1}(\Omega)\right)^{2}$ and $\nabla \cdot \mathrm{w}=0$ in $\Omega$ there exists $\psi \in H_{0}^{2}(\Omega)$ such that $\mathrm{w}=\operatorname{rot} \psi$. In $\left[5\right.$, thm. 3.6] it is proven that there exists $\psi_{N} \in H_{0}^{2}(\Omega)$ such that $\psi_{N}^{i} \in P_{N}\left(\bar{\Omega}_{i}\right), \psi_{N}^{i}=\psi_{N}^{i+1}$ on $\Gamma_{i}$ and $\frac{\partial \psi_{N}^{i}}{\partial \mathbf{n}^{i}}=\frac{\partial \psi_{N}^{i+1}}{\partial \mathbf{n}^{i}}$ on $\Gamma_{i}$ for $i=1, \ldots, M-1$ which verifies

$$
\left\|\psi-\psi_{N}\right\|_{2} \leq C \sum_{i=1}^{M} N^{2-\mu_{i}}\left\|\psi^{i}\right\|_{\mu_{i}, i}, \quad \mu_{i} \geq 2 .
$$

Here $C$ is a positive constant independent of $N$ and $\psi$. Defining $\mathbf{w}_{N}=\operatorname{rot} \psi_{N}$ we have that $\mathbf{w}_{N} \in V_{N}$ and

$$
\left\|\mathrm{w}-\mathrm{w}_{N}\right\|_{1} \leq\left\|\psi-\psi_{N}\right\|_{2} \leq C \sum_{i=1}^{M} N^{2-\mu_{i}}\left\|\psi^{i}\right\|_{\mu_{i}, i} \leq C \sum_{i=1}^{M} N^{2-\mu_{i}}\left\|\mathbf{w}^{i}\right\|_{\mu_{i-1}, i}
$$

for $\mu_{i} \geq 2$. This gives $(2.1)$ for $\sigma_{i} \geq 1$.

Theorem 2.2. Let $u$ and $u_{N}$ be the solutions of problems (1.2) and (1.4) respectively. If $\mathrm{u}^{i} \in\left(H^{\sigma_{i}}\left(\Omega_{i}\right)\right)^{2}, \sigma_{i} \geq 1$ and $\mathrm{f} \in\left(H^{\mu_{i}}\left(\Omega_{i}\right)\right)^{2}, \mu_{i} \geq 1$, for $i=1, \ldots, M$, then there exists a positive constant $C$ independent of $N$, $\mathbf{u}$, and $f$ such that

$$
\left\|\mathrm{u}-\mathrm{u}_{N}\right\|_{1} \leq C \sum_{i=1}^{M}\left\{N^{1-\sigma_{i}}\left\|\mathbf{u}^{i}\right\|_{\sigma_{i}, i}+N^{1-\mu_{i}}\left\|\mathbf{f}^{i}\right\|_{\mu_{i}, i}\right\}
$$


Proof. From a general approximation result (see e.g. [3, Corollary II.3]) we have

$$
\begin{aligned}
&\left\|\mathbf{u}-\mathbf{u}_{N}\right\|_{1} \leq C\left\{\operatorname { i n f } _ { \mathbf { w } _ { N } \in V _ { N } , \nabla \cdot \mathbf { w } _ { N } = 0 } \left\{\left\|\mathbf{u}-\mathbf{w}_{N}\right\|_{1}+\right.\right. \\
&\left.\sup _{\mathbf{s}_{N} \in V_{N}} \frac{\left(\left(\nabla \mathbf{w}_{N}, \nabla \mathbf{z}_{N}\right)\right)-\left(\left(\nabla \mathbf{w}_{N}, \nabla \mathbf{z}_{N}\right)\right)_{N}}{\left\|\nabla \mathbf{z}_{N}\right\|}\right\}+ \\
&\left.\sup _{\mathbf{N} \in V_{N}} \frac{\left(\left(\mathbf{f}, \mathbf{z}_{N}\right)\right)-\left(\left(\mathbf{f}, \mathbf{z}_{N}\right)\right)_{N}}{\left\|\nabla \mathbf{z}_{N}\right\|}\right\},
\end{aligned}
$$

where $C$ is a positive constant related to the constant $C_{1}$ appearing in (0.5). Choosing $\mathrm{w}_{N}=\Pi_{N-1}^{1, d i v} \mathrm{u}$ the first term at the right hand side of $(2.4)$ can be estimated using (2.1). Moreover, since the Legendre Gauss Lobatto quadrature formula (see (0.1)) is exact on $P_{2 N-1}\left(\bar{\Omega}_{i}\right)$, the second term vanishes. The last term in (2.4) is estimated by using known results on the interpolation error (see [8, section 9.4.3]).

Our aim is now to prove an error estimate for the pressure. We remark that if $M_{N}$ is given by (1.12) then $p_{N}$ vanishes at the comer points $a_{0}, b_{0}, a_{M}, b_{M}$. On the other hand the solution $p$ of the continuous problem do not vanishes at these points. Hence if $M_{N}$ is defined by (1.12) the spectral accuracy cannot be achieved. Using an idea introduced in [6] we now define a new pressure space $\tilde{M}_{N}$. The following is a direct consequence of theorem 1.2 .

Proposition 2.1. Let $\tilde{M}_{N}$ be a subspace of $\prod_{i=1}^{M} P_{N}\left(\bar{\Omega}_{i}\right)$ such that

$$
Q_{N, \lambda}:=\left\{q \in\left\{\prod_{i=1}^{M} P_{\{\lambda N \mid}\left(\bar{\Omega}_{i}\right)\right\} / R: q^{i}=q^{i+1} \text { at } a_{i} \text { and } b_{i}, i=1, \ldots, M-1\right\} \subset \bar{M}_{N}
$$

where $\lambda \in(0,1)$ and $[\lambda N]$ is the integer part of $\lambda N$. Moreover suppose that there exist an isomorphism $G_{N}: M_{N} \rightarrow \tilde{M}_{N}$ such that for any $q \in M_{N}$

$$
((q, \nabla \cdot \mathrm{v}))_{N}=\left(\left(G_{N} q, \nabla \cdot \mathrm{v}\right)\right)_{N} \quad \forall \mathbf{v} \in V_{N}
$$

and

$$
\left\|G_{N} q\right\| \leq C\|q\|
$$

with a constant $\mathrm{C}$ independent of $q$ and $N$. Then the inf-sup condition (1.13) holds with $M_{N}$ substituted by $\tilde{M}_{N}$.

A new discrete problem is obtained by using $\tilde{M}_{N}$ instead of $M_{N}$ in (1.4). From the proposition 2.1 it follows that such problem has a unique solution $\left(\tilde{\mathbf{u}}_{N}, \tilde{p}_{N}\right)$ and that $\tilde{\mathbf{u}}_{N}=\mathbf{u}_{N}$. In other words the discrete velocity do not depends on the choice of the pressure space provided the assumptions (2.5)-(2.7) hold. We now give an example of pressure space.

Given an integer $i, 1 \leq i \leq M$, and a point $\mathrm{P} \in \Xi_{N}^{i}$, the characteristic function $\chi_{\mathrm{P}}^{i}$ (defined in (0.6)) can be written in the form

$$
\chi_{\mathrm{P}}^{i}=\sum_{k=0}^{N} \sum_{l=0}^{N} \alpha_{k, l}^{i} L_{k}^{i}(x) L_{l}(y) .
$$


We define $\tilde{\chi}_{P}^{i}$ by

$$
\tilde{\chi}_{\mathrm{P}}^{i}=\sum_{k=[\lambda N]+1}^{N-1} \sum_{l=[\lambda N]+1}^{N-1} \alpha_{k, l}^{i} L_{k}^{i}(x) L_{l}(y),
$$

and we consider the space:

$$
\begin{aligned}
\tilde{M}_{N}=\{ & q \in \prod_{i=1}^{M} P_{N}\left(\tilde{\Omega}_{i}\right): \\
& \left(q^{1}, \tilde{\chi}_{a_{0}}^{1}\right)_{N, 1}=\left(q^{1}, \tilde{\chi}_{b_{0}}^{1}\right)_{N, 1}=\left(q^{M}, \tilde{\chi}_{a_{M}}^{M}\right)_{N, M}=\left(q^{M}, \tilde{\chi}_{b_{M}}^{M}\right)_{N, M}=0, \\
& q^{i}\left(a_{i}\right)=q^{i+1}\left(a_{i}\right), q^{i}\left(b_{i}\right)=q^{i+1}\left(b_{i}\right), i=1, \ldots, M-1, \\
& \sum_{i=1}^{M}\left(q^{i}, 1\right)_{N, i}=0, \sum_{i=1}^{M}\left(q^{i}, L_{N}(y)\right)_{N, i}=0, \\
& \left.\left(q^{i}, L_{N}^{i}(x)\right)_{N, i}=0,\left(q^{i}, L_{N}^{i}(x) L_{N}(y)\right)_{N, i}=0, i=1, \ldots, M\right\} .
\end{aligned}
$$

Recalling (1.12) we remark that the difference between $M_{N}$ and $\bar{M}_{N}$ consists in the first four conditions appearing in (2.10). From (2.8)-(2.10) it is clear that $\tilde{M}_{N}$ verifies (2.5). We now prove the following.

Proposition 2.2 If $\tilde{M}_{N}$ is given by (2.10) then there exists an isomorphism $G_{N}$ such that (2.6) and (2.7) hold.

Proof. Let us denote by $\chi_{k}, k=1, \ldots, 4$ the four elements of $\prod_{i=1}^{M} P_{N}\left(\bar{\Omega}_{i}\right)$ given in (1.6)(1.7) and by $\tilde{\chi}_{k}, k=1, \ldots, 4$ the corresponding elements with $\chi_{\mathrm{P}}^{i}$ substituted by $\tilde{\chi}_{\mathrm{P}}^{i}(\operatorname{see}(2.9))$. We define $\bar{\chi}_{k}, k=1, \ldots, 4$ as the projection, for the inner product $((\cdot, \cdot))_{N}$, of $\chi_{k}$ on the subspace of $\prod_{i=1}^{M} P_{N}\left(\widetilde{\Omega}_{i}\right)$ which is orthogonal to the elements given in (1.8)-(1.10).

For any $q \in M_{N}$ we define

$$
G_{N} q=q-\sum_{k=1}^{4} \frac{\left(\left(q, \tilde{\chi}_{k}\right)\right)_{N}}{\left\|\tilde{\chi}_{k}\right\|_{N}^{2}} \bar{\chi}_{k}
$$

From the definition of $\bar{\chi}_{k}, k=1, \ldots, 4$ we have that $G_{N} q$ is orthogonal to the elements (1.8)-(1.10) for any $q \in M_{N}$. Moreover using the expansion of $\chi_{k}, \tilde{\chi}_{k}, \bar{\chi}_{k}$, $1 \leq k \leq 4$, in Legendre series (see e.g. [6, Prop.V.3]) one can show that

$$
\left(\left(\bar{\chi}_{k}, \tilde{\chi}_{l}\right)\right)_{N}=\left\|\tilde{\chi}_{l}\right\|_{N}^{2} \delta_{k, l} \text { and }\left\|\bar{\chi}_{k}\right\|_{N} /\left\|\tilde{\chi}_{k}\right\|_{N} \leq C \quad k, l=1, \ldots, 4,
$$

where $\delta_{k, l}$ is the Kronecher symbol. Using (2.11) it follows that $G_{N} q$ is orthogonal to $\tilde{\chi}_{l}, l=1, \ldots, 4$, hence $G_{N} q \in \tilde{M}_{N}$ for any $q \in M_{N}$.

Since $\bar{\chi}_{k} \in Z_{N, M} k=1, \ldots, 4$ we deduce from proposition 1.2 that $(2.6)$ holds. To prove (2.7) we remark that using the Cauchy-Schwarz inequality and (2.12) we have

$$
\left\|G_{N} q\right\|_{N} \leq\|q\|_{N}\left(1+\sum_{k=1}^{4} \frac{\left\|\bar{\chi}_{k}\right\|_{N}}{\left\|\tilde{\chi}_{k}\right\|_{N}}\right) \leq C\|q\|_{N}
$$


Hence (2.7) follows from (0.5).

We can now give a convergence estimate for the pressure.

Theorem 2.9. Let $\left(\mathrm{u}_{N}, \tilde{p}_{N}\right)$ be the solution of problem (1.4) with $M_{N}$ substituted by $\tilde{M}_{N}$. If $(\mathbf{u}, p)$ is the solution of problem (1.2) and $\mathbf{u}^{i} \in\left(H^{\sigma_{i}}\left(\Omega_{i}\right)\right)^{2}$, $p \in H^{\sigma_{i}-1}\left(\Omega_{i}\right) \cap H^{1}(\Omega), \sigma_{i} \geq 2, \mathrm{f} \in\left(H^{\mu_{i}}\left(\Omega_{i}\right)\right)^{2}, \mu_{i} \geq 1,1 \leq i \leq M$, then we have

$$
\left\|p-\tilde{p}_{N}\right\|_{0} \leq C\left\{\sum_{i=1}^{M} N^{2-\sigma_{i}}\left\{\left\|\mathbf{u}^{i}\right\|_{\sigma_{i}, i}+\left\|p^{i}\right\|_{\sigma_{i}-1, i}\right\}+\sum_{i=1}^{M} N^{2-\mu_{i}}\left\|f^{i}\right\|_{\mu_{i}, i}\right\},
$$

where $C$ is a positive constant independent of $N, p, \mathbf{u}$ and $\mathbf{f}$.

Proof. From a classical approximation result (see e.g. [3, corollary 2.3]) and using theorems 1.2, 2.1 and proposition 2.1 we obtain

$$
\left\|p-\tilde{p}_{N}\right\|_{0} \leq C N\left\{\inf _{q N \in \tilde{M}_{N}}\left\|p-q_{N}\right\|_{0}+\sum_{i=1}^{M}\left\{N^{1-\sigma_{i}}\left\|\mathbf{u}^{i}\right\|_{\sigma_{i}, i}+N^{1-\mu_{i}}\left\|f^{i}\right\|_{\mu_{i}, i}\right\}\right\} .
$$

Recalling (2.5) and an approximation result due to Bernardi and Maday (see [5 thm. III.3]), we obtain

$$
\inf _{q_{N} \in \tilde{M}_{N}}\left\|p-q_{N}\right\|_{0} \leq \inf _{q_{N} \in Q_{N, \lambda}}\left\|p-q_{N}\right\|_{0} \leq C \sum_{i=1}^{M} N^{1-\sigma_{i}}\left\|p^{i}\right\|_{\sigma_{i}, i},
$$

hence (2.13) follows from (2.14) and (2.15).

Remark 2.1. Let $\left(\mathrm{u}_{N}, p_{N}\right)$ be the solution of problem (1.4), and let $\left(\mathrm{u}_{N}, \tilde{p}_{N}\right)$ be the solution of the same problem with $M_{N}$ replaced by $\vec{M}_{N}$. Recalling (2.11)(1.4) and (2.6) it is readly seen that

$$
\tilde{p}_{N}=G_{N} p_{N} .
$$

Hence to obtain a spectral approximation of the pressure one can either solve (1.4) and compute $\tilde{p}_{N}$ from (2.16), or solve directly (1.4) with $M_{N}$ replaced by $\tilde{M}_{N}$. 


\section{Plurirectangular Domains.}

In this section we consider a plurirectangle $\Omega$ partitioned in rectangles $\Omega_{i}, i=$ $1, \ldots, M$, where $M$ is a fixed integer. We assume that the decomposition of $\Omega$ is such that the intersection of two adiacent subdomains is either a point or an entire edge.

The finite dimensional space $V_{N}$ is defined again by (1.3) and the discrete problem is given by (1.4). The crucial difference with the case of aligned subdomains lies in the choice of the pressure space $M_{N}$. Let us first consider, as an example, the domain of figure 3.1 .

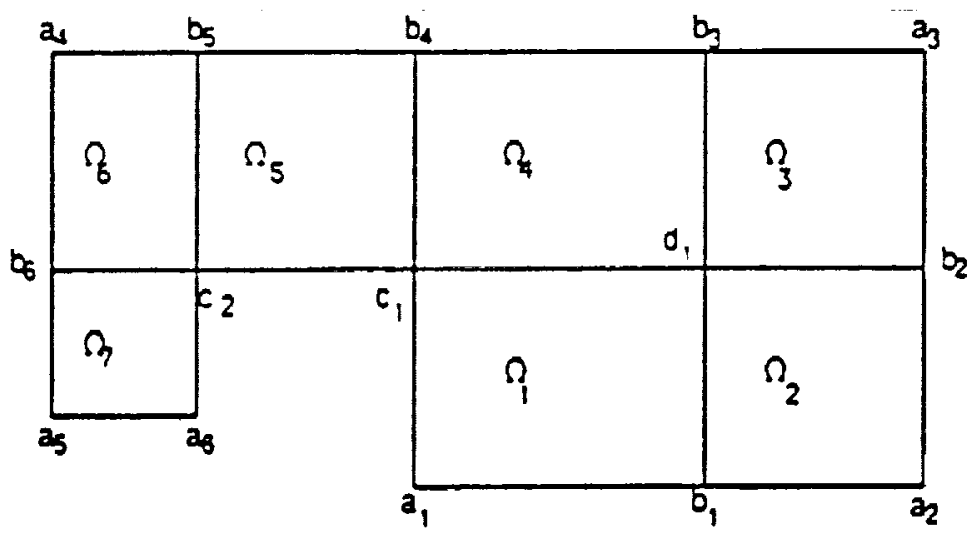

\section{figưre 3.1}

The points $a_{i}, i=1, \ldots, 6$, are the non-reentrant corners of $\partial \Omega ; b_{i}, i=1, \ldots, 6$, are the points of $\partial \Omega$ which are the intersection of two subdomains, $c_{1}$ and $c_{2}$ are the reentrant corners of $\partial \Omega$ and $d_{1}$ is a so called "cross point", i.e. the intersection of four subdomains.

Following the lines of section one we define $M_{N}$ as the subspace of $\prod_{i=1}^{7} P_{N}\left(\Omega_{i}\right)$ whose elements are orthogonal to $Z_{N, 7}$ (see (1.5) and (1.11)). In this case there are 30 independent elements of $Z_{N, 7}$, and the space $M_{N}$ is given by

$$
\begin{aligned}
M_{N}=\{ & q \in \prod_{i=1}^{7} P_{N}\left(\Omega_{i}\right): q=0 \text { in } a_{i}, i=1, \ldots, 6, \\
& q^{i}=q^{i+1} \text { in } b_{i}, i=1, \ldots, 6, \\
& q^{1}+q^{5}=q^{4} \text { in } c_{1}, q^{5}+q^{7}=q^{6} \text { in } c_{2}, \\
& q^{1}+q^{3}=q^{2}+q^{4} \text { in } d_{1}, \\
& \sum_{i=1}^{7}\left(q^{i}, 1\right)_{N, i}=0, \\
& \left(\left(q^{i}, L_{N}^{i}(x) L_{N}^{i}(y)\right)_{N, i}=0 \quad i=1, \ldots, 7,\right. \\
& \left(q^{7}, L_{N}^{7}(x)\right)_{N, 7}=0, \sum_{i=1}^{2}\left(q^{i}, L_{N}^{i}(x)\right)_{N, i}=0, \sum_{i=3}^{6}\left(q^{i}, L_{N}^{i}(x)\right)_{N, i}=0,
\end{aligned}
$$




$$
\begin{aligned}
& \sum_{i=6}^{7}\left(q^{i}, L_{N}^{i}(y)\right)_{N, i}=0,\left(q^{5}, L_{N}^{5}(y)\right)_{N, 5}=0,\left(q^{1}, L_{N}^{1}(y)\right)_{N, 1}+\left(q^{4}, L_{N}^{4}(y)\right)_{N, 4}=0 \\
& \left.\sum_{i=2}^{3}\left(q^{i}, L_{N}^{i}(y)\right)_{N, i}=0\right\} .
\end{aligned}
$$

The first 15 conditions are orthogonality relations, as a matter of fact they can be written as follows.

$$
\begin{aligned}
& \left(q^{i}, \chi_{a_{i}}^{i}\right)_{N, i}=0, i=1,2,3,\left(q^{6}, \chi_{a_{6}}^{6}\right)_{N, 6}=\left(q^{7}, \chi_{a_{5}}^{7}\right)_{N, 7}=\left(q^{7}, \chi_{a_{6}}^{7}\right)_{N, 7} \\
& \left(q^{i}, \chi_{b_{i}}^{i}\right)_{N, i}=\left(q^{i+1}, \chi_{b_{i}}^{i+1}\right)_{N, i}, i=1, \ldots, 6 \\
& \left(q^{1}, \chi_{c_{1}}^{1}\right)_{N, 1}+\left(q^{5}, \chi_{c_{1}}^{5}\right)_{N, 5}=\left(q^{4}, \chi_{c_{1}}^{4}\right)_{N, 4}, \\
& \left(q^{5}, \chi_{c_{2}}^{5}\right)_{N, 5}+\left(q^{7}, \chi_{c_{2}}^{7}\right)_{N, 7}=\left(q^{6}, \chi_{c_{2}}^{6}\right)_{N, 6}, \\
& \left(q^{1}, \chi_{d_{1}}^{1}\right)_{N, 1}+\left(q^{3}, \chi_{d_{1}}^{3}\right)_{N, 3}=\left(q^{2}, \chi_{d_{1}}^{2}\right)_{N, 2}+\left(q^{4}, \chi_{d_{1}}^{4}\right)_{N, 4} .
\end{aligned}
$$

These conditions will be called "physical conditions" as opposite to the last 15 conditions appearing in (3.1) which will be called "spectral".

We now suppose that $\Omega$ is any plurirectangle partitioned in $M$ rectangles $\Omega_{i}$, $i=1, \ldots, M$. Let $M_{N}$ be defined by (1.11), in the following we describe the conditions which characterize the elements of $M_{N}$.

A physical condition is associated to every node of the decomposition. Precisely if $\mathrm{P}$ is a non-reentrant corner of $\partial \Omega$ (as $a_{i}, i=1, \ldots, 6$ in fig.3.1) then we impose that any $q \in M_{N}$ vanishes at $\mathrm{P}$. If $\mathrm{P}$ is a reentrant corner (see fig. 3.2 ) then we impose $q^{k}(\mathrm{P})+q^{n}(\mathrm{P})=q^{l}(\mathrm{P})$

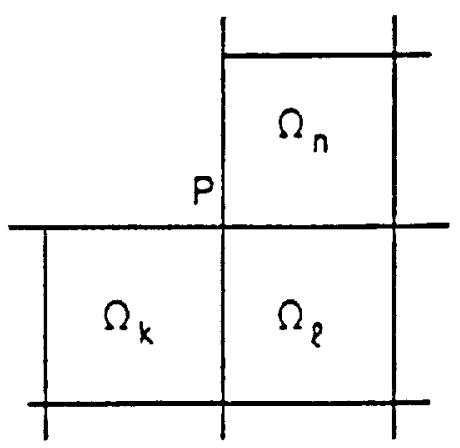

figure 3.2

If a node $\mathrm{P}$ belongs to $\partial \Omega$ and it is not a corner (as $b_{i}, i=1, \ldots, 6$ in fig.3.1) we impose the continuity of $q$ at $P$. Finally if $P$ is a cross point (see fig.3.3) we impose the "continuity" condition $q^{m}(\mathrm{P})+q^{l}(\mathrm{P})=q^{k}(\mathrm{P})+q^{n}(\mathrm{P})$.

Let us now describe the spectral conditions appearing in the definition of $M_{N}$. As in the continuous case we impose that any $q \in M_{N}$ is orthogonal to the constants. The 


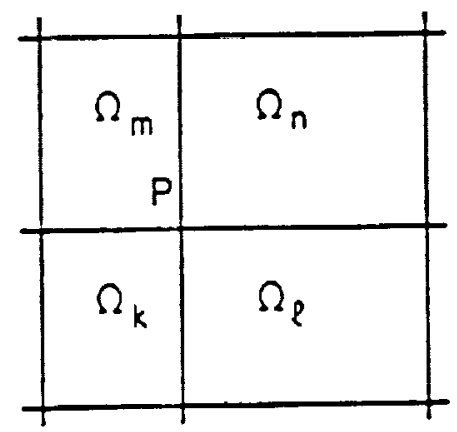

figure 3.3

orthogonality of $q$ to $L_{N}^{i}(x) L_{N}^{i}(y), i=1, \ldots, M$, gives $M$ additional conditions. Let us now call "horizontal strip" any maximal set of horizontally aligned subdomains. For instance in figure 3.1 these strips are $\left\{\Omega_{7}\right\},\left\{\Omega_{1}, \Omega_{2}\right\}$ and $\left\{\Omega_{i}, i=3, \ldots, 6\right\}$. If a horizontal strip is given by $\left\{\Omega_{l}, l \in \Delta\right\}$ where $\Lambda$ is an index set, then we impose that $q$ is orthogonal to the function which equals $L_{N}^{l}(y)$ on $\Omega_{l}, l \in \Lambda$, and vanishes on the other subdomains. Similarly if $\left\{\Omega_{k}, k \in K\right\}$ is a "vertical strip" (these strips are $\left\{\Omega_{6}, \Omega_{7}\right\},\left\{\Omega_{5}\right\},\left\{\Omega_{1}, \Omega_{4}\right\}$ and $\left\{\Omega_{2}, \Omega_{3}\right\}$ in fig.3.1) then we impose that $q$ is orthogonal to the function which equals $L_{N}^{k}(x)$ on $\Omega_{k}, k \in K$, and vanishes on the other subdomains.

Using this definition of the pressure space $M_{N}$, the "inf-sup condition" (1.13) holds also in the case of a plurirectangle (see remark A.1). In particular this implies that the discrete problem is well posed. Moreover, it is readly seen that if $\mathbf{v}_{N} \in V_{N}$ then $\nabla \cdot \mathbf{v}_{N} \in M_{N}$, so that the discrete solution is divergence-free for any value of the discretization parameter.

We now give the collocation form of problem (1.4) for a decomposition of a plurirectangle $\Omega$.

Let $\left(\mathbf{u}_{N}, p_{N}\right)$ be the solution of (1.4), we have

$$
-\Delta \mathrm{u}_{N}^{i}(\xi)+\nabla p_{N}^{i}(\xi)=f(\xi), \quad \forall \Xi_{N}^{i} \cap \Omega_{i}, i=1, \ldots, M .
$$

Denoting by $\Gamma_{k, l}$ the common side of two subdomains $\Omega_{k}, \Omega_{l}$, (not including the end-points) we have

$$
\begin{aligned}
& \left\{\frac{\partial \mathbf{u}_{N}^{k}}{\partial \mathbf{n}^{k}}-p_{N}^{k} \mathbf{n}^{k}\right\}(\xi)-\left\{\frac{\partial \mathbf{u}_{N}^{l}}{\partial \mathbf{n}^{k}}-p_{N}^{l} \mathbf{n}^{k}\right\}(\xi)= \\
& =-\left\{-\Delta \mathbf{u}_{N}^{k}+\nabla p_{N}^{k}-\mathbf{f}^{k}\right\}(\xi) \omega_{N}^{k}-\left\{-\Delta \mathbf{u}_{N}^{l}+\nabla p_{N}^{l}-\mathbf{f}^{l}\right\}(\xi) \omega_{N}^{l}, \quad \forall \xi \in \Gamma_{k, l} .
\end{aligned}
$$

Let $\xi$ be a cross-point and consider, for simplicity the notation of figure 3.4 . Setting

$$
D^{i}\left(\mathrm{u}_{N}^{i}, p_{N}^{i}\right)(\xi)=\sum_{k=1}^{2}\left\{\frac{\partial \mathrm{u}_{N}^{i}}{\partial \mathrm{n}^{i, k}}-p_{N}^{i} \mathrm{n}^{i, k}\right\}(\xi)
$$




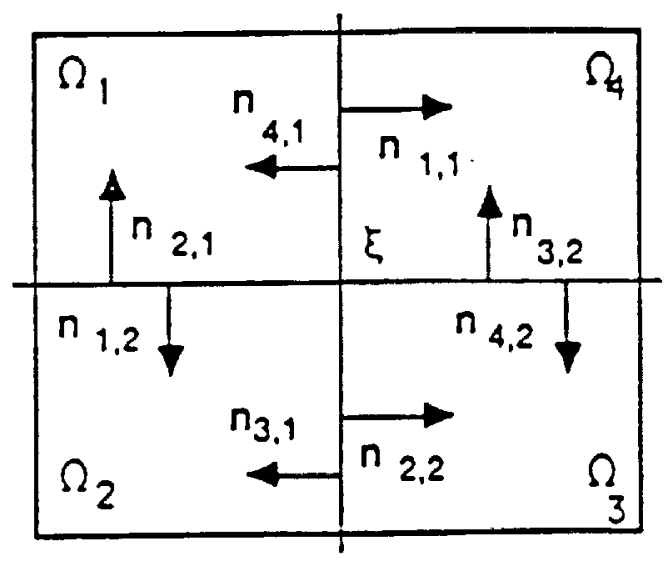

figure 3.4

the cross-point condition is

$$
\sum_{i=1}^{4} D^{i}\left(\mathrm{u}_{N}^{i}, p_{N}^{i}\right)(\xi)=-\sum_{i=1}^{4}\left\{-\Delta \mathrm{u}_{N}^{i}+\nabla p_{N}^{i}-\mathrm{f}^{i}\right\}(\xi) \omega_{N}^{i}, \quad \forall \text { cross - point } \xi
$$

Finally $\mathrm{u}_{N}$ verifies the incompressibility condition, i.e.

$$
\nabla \cdot u_{N}^{i}=0 \quad \text { in } \Xi_{N}^{i}, i=1, \ldots, M .
$$

Actually, it is sufficient to impose (3.6) in a subset of $\left\{\Xi_{N}^{i}, i=1, \ldots, M\right\}$. Precisely, since $\mathbf{u}_{N}=0$ on $\partial \Omega$ then $\nabla \cdot \mathbf{u}_{N}$ automatically vanishes at any non-reentrant corner of $\partial \Omega$. If $\mathrm{P}$ is a node on $\partial \Omega$ which is not a corner (e.g. $b_{1}$ in figure 4.1) it is sufficient to impose only one condition at $P$ (e.g. for fig. $4.1 \nabla \cdot \mathbf{u}_{N}^{1}\left(b_{1}\right)=0$ implies $\nabla \cdot \mathbf{u}_{N}^{2}\left(b_{1}\right)=0$ since $\left.\nabla \cdot \mathbf{u}_{N} \in M_{N}\right)$. Similarly if $\mathrm{P}$ is given in figure 3.2 or 3.3 it is sufficient to impose $\nabla \cdot \mathbf{u}_{N}^{k}=\nabla \cdot \mathbf{u}_{N}^{n}=0$ and $\nabla \cdot \mathbf{u}_{N}^{k}=\nabla \cdot \mathbf{u}_{N}^{n}=\nabla \cdot \mathbf{u}_{N}^{m}=0$ at $P$ respectively. It is also not necessary to impose (3.6) at some collocation points internal to each subdomain (this follows from the fact that $\nabla \cdot \mathbf{u}_{N} \in M_{N}$ and hence it verifies the spectral conditions). Precisely one can eliminate in (3.6) one point for each subdomain, one point for each horizontal strip, one point for each vertical strip and one point choosen in an arbitrary subdomain. The points which are eliminated must verify some hypothesis of the kind of (1.8),(1.9); if in every subdomain these points are not aligned then these hypothesis hold. Summarizing, (3.6) reduces to

$$
\nabla \cdot \mathrm{u}_{N}^{i}=0 \quad \text { in } \Xi_{N}^{i} / \Lambda_{N, M}, i=1, \ldots, M
$$

where $\Lambda_{N, M}$ is a set of $n$ collocation points and $n$ is the codimension of $M_{N}$ in $\prod_{i=1}^{M} P_{N}\left(\bar{\Omega}_{i}\right)$.

The velocity error estimate (2.3) holds also in the case of a plurirectangle. The proof works exactly in the same way.

As in the case of the aligned subdomains to obtain an error estimate for the pressure one has to modify the space $M_{N}$ introducing a new space $\tilde{M}_{N}$ such that 
the thesis of proposition 2.1 holds. Precisely the physical conditions related to the corners of $\partial \Omega$ have to be removed. In the case of a non-reentrant corner $P \in \partial \Omega_{i}$ the condition $q^{i}(\mathrm{P})=0$ has to be substituted by $\left(q^{i}, \tilde{\chi}_{\mathrm{P}}^{i}\right)_{N, i}=0$ (as in the case of the aligned subdomains, see (2.8)-(2.10)). On the other hand if $P$ is a reentrant corner (see fig. 3.2) the condition $q^{k}+q^{n}=q^{l}$ at $\mathrm{P}$ has to be substituted by $\left(q^{k}, \tilde{\chi}_{\mathrm{P}}^{k}\right)_{N, k}+\left(q^{n}, \tilde{\chi}_{\mathrm{P}}^{n}\right)_{N, n}=\left(q^{l}, \tilde{\chi}_{\mathrm{P}}^{l}\right)_{N, l}$. If the other conditions of $M_{N}$ are not changed we obtain a new space $\tilde{M}_{N}$ which verifies (2.6),(2.7) and:

$\left\{q \in\left\{\prod_{i=1}^{M} P_{[\lambda N]}\left(\bar{\Omega}_{i}\right\} / \mathrm{R}: q\right.\right.$ is continuous at the verteces of the subdomains $\} \subset \tilde{M}_{N}$

Then the result of proposition 2.1 holds and the estimate (2.13) can be obtained with exactly the same proof technique. 


\section{Appendix}

In this section we prove proposition 1.1 and theorem 1.2. We first consider two aligned subdomains, the proof in the general case will follow from an induction argument. Let us precise some notation. The outward normal unit vector to $\partial \Omega$ is denoted by $\mathrm{n}$. For $i=1,2$ we denote by $\tau^{i}$ the counterclockwise unit tangent vector to $\partial \Omega_{i}$ and by $\mathrm{n}^{i}$ the outward normal unit vector to $\partial \Omega_{i}$. The notation $\tau_{j}^{i}, \mathbf{n}_{j}^{i}, i, j=1,2$, are introduced in figure A.1.

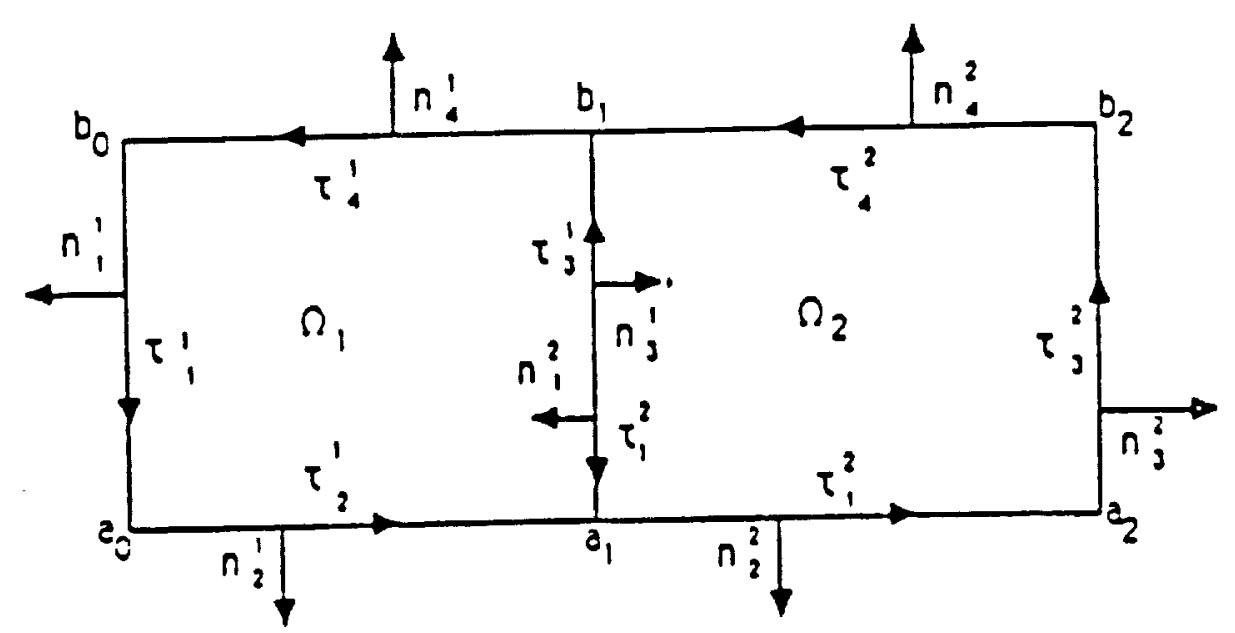

fig. A.1 The decomposition of $\Omega$ with $M=2$.

Recalling the definition (0.6) we set for any $n \geq 1$ :

$$
\begin{aligned}
& J_{n}=\frac{L_{n+1}-L_{n-1}}{2 n+1} \\
& J_{n}^{i}=\frac{x_{i}-x_{i-1}}{2} \cdot \frac{L_{n+1}^{i}-L_{n-1}^{i}}{2 n+1}, \text { for } i=1,2,
\end{aligned}
$$

where $\left\{x_{i}\right\}$ are the abscisses of the points $\left\{a_{i}\right\}$ for $i=0,1,2$. The polynomials $J_{n}$ and $J_{n}^{i}$ are the primitive of $L_{n}$ and $L_{n}^{i}$ respectively, i.e.

$$
\left\{\begin{array}{l}
J_{n}^{\prime}=L_{n}, \quad J_{n}(-1)=J_{n}(1)=0 \\
\left(J_{n}^{i}\right)^{\prime}=L_{n}^{i}, \quad J_{n}^{i}\left(x_{i-1}\right)=J_{n}^{i}\left(x_{i}\right)=0, \quad \text { for } i=1,2,
\end{array}\right.
$$

Moreover we have for any $n \geq 1$ and $i=1,2$ :

$$
\left\{\begin{array}{l}
\left\|L_{n}^{i}\right\|_{0, i}^{2}=\frac{C_{1}}{2 n+1} \\
\frac{C_{2}}{(2 n+1)^{3}} \leq\left\|J_{n}^{i}\right\|_{0, i}^{2} \leq \frac{C_{3}}{(2 n+1)^{3}}
\end{array},\right.
$$


where $C_{1}, C_{2}$ and $C_{3}$ are positive constants independent of $n$.

Let us denote by $\tilde{Z}_{N, 2}$ the subspace of $P_{N}\left(\bar{\Omega}_{1}\right) \times P_{N}\left(\bar{\Omega}_{2}\right)$ spanned by the 12 elements

$$
\begin{gathered}
\left(\chi_{a_{0}}^{1}, 0\right), \quad\left(\chi_{b_{0}}^{1}, 0\right), \quad\left(0, \chi_{a_{3}}^{2}\right), \quad\left(0, \chi_{b_{2}}^{2}\right), \\
\left(\chi_{a_{1}}^{1},-\chi_{a_{1}}^{2}\right), \quad\left(\chi_{b_{1}}^{1},-\chi_{b_{1}}^{2}\right), \\
(1,1), \quad\left(L_{N}(y), L_{N}(y)\right) \\
\left(L_{N}^{1}(x), 0\right), \quad\left(0, L_{N}^{2}(x)\right), \quad\left(L_{N}^{1}(x), L_{N}(y), 0\right), \quad\left(0, L_{N}^{2}(x) L_{N}(y)\right) .
\end{gathered}
$$

These are precisely the spurious modes given in (1.6)-(1.10) for two subdomains. Using $(0.2)$ it is readly seen that $\tilde{Z}_{N, 2}$ is contained in the space $Z_{N, 2}$ defined in (1.5). In the sequel we prove that, actually, $\tilde{Z}_{N, 2}$ and $Z_{N, 2}$ coincide.

Let $q \in P_{N}\left(\bar{\Omega}_{1}\right) \times P_{N}\left(\bar{\Omega}_{2}\right)$ be orthogonal, for the inner product $(0.4)$, to $\tilde{Z}_{N, 2}$ (i.e. to the elements (A.3)-(A.6)). For $i=1,2, q^{i}$ can be written in the form

$$
q^{i}=\sum_{0 \leq k, l \leq N-1} \alpha_{k l}^{i} L_{k}^{i} \otimes L_{l}+\sum_{k=0}^{N-1} \beta_{k}^{i} L_{k}^{i} \otimes J_{N-1}+\sum_{l=1}^{N-1} \gamma_{l}^{i} J_{N-1}^{i} \otimes L_{l} .
$$

Let us define two continuous functions $A$ and $B:\left[x_{0}, x_{2}\right] \rightarrow \mathbf{R}$ such that

$$
A \text { and } B \text { are linear in }\left[x_{i-1}, x_{i}\right], \quad i=1,2
$$

$$
A\left(x_{0}\right)=B\left(x_{0}\right)
$$

and

$$
\forall x \in] x_{i-1}, x_{i}\left[\quad A \prime(x)=\alpha_{00}^{i} \quad B \prime(x)=\beta_{0}^{i} \text { for } i=1,2 .\right.
$$

We define the function $\mathrm{v}=\left(v_{1}, v_{2}\right) \in\left(P_{N}\left(\bar{\Omega}_{1}\right) \times P_{N}\left(\bar{\Omega}_{2}\right)\right)^{2}$ as follows:

$$
\begin{aligned}
& v_{1}^{i}=\sum_{0 \leq l \leq k \leq N-1, k+l \neq 0} \alpha_{k l}^{i} J_{k}^{i} \otimes J_{l}+\sum_{k=1}^{N-1} \beta_{k}^{i} J_{k}^{i} \otimes J_{N-1}+A^{i} \otimes L_{0}+B^{i} \otimes J_{N-1}, \\
& v_{2}^{i}=\sum_{0 \leq k \leq l \leq N-1} \alpha_{k l}^{i} L_{k}^{i} \otimes J_{l}+\sum_{l=1}^{N-1} \gamma_{l}^{i} J_{N-1}^{i} \otimes J_{l} \quad \text { for } i=1,2 .
\end{aligned}
$$

Lemma A.1. Let $q \in P_{N}\left(\bar{\Omega}_{1}\right) \times P_{N}\left(\bar{\Omega}_{2}\right)$ be orthogonal to $\tilde{Z}_{N, 2}$ for the inner product $((\cdot, \cdot))_{N}$ defined in (0.4). If $q$ is written in the form (A.7) and $v$ is given by (A.11) then we have

$$
\begin{gathered}
\nabla \cdot \mathrm{v}^{i}=q^{i} \quad \text { for } i=1,2, \\
\mathbf{v} \cdot \mathrm{n}=0 \quad \text { on } \partial \Omega \\
\mathrm{v}^{1} \cdot \mathrm{n}_{3}^{1}=-\mathrm{v}^{2} \cdot \mathrm{n}_{1}^{2} \quad \text { on } \Gamma_{1} .
\end{gathered}
$$


Proof. From (A.1) (A.7) and (A.11) we obtain (A.12). Using (A.1) we deduce that $\mathbf{v} \cdot \mathbf{n}=0$ on the two segments $\left[a_{0}, a_{2}\right]$ and $\left[b_{0}, b_{2}\right]$. Moreover we remark that the orthogonality of $q$ to the elements given in (A.5) reads as follows

$$
\sum_{i=1}^{2} \alpha_{00}^{i}\left(x_{i}-x_{i-1}\right)=\sum_{i=1}^{2} \beta_{0}^{i}\left(x_{i}-x_{i-1}\right)=0 .
$$

From (A.8)-(A.10) we deduce

$$
A\left(x_{2}\right)=B\left(x_{2}\right)=0 \text {. }
$$

Then the condition $\mathbf{v} \cdot \mathbf{n}=0$ on the two segments $\left[a_{0}, b_{0}\right]$ and $\left[a_{2}, b_{2}\right]$ follows from (A.1)(A.9) and (A.15). Finally since $A$ and $B$ are continuous we deduce (A.14) from (A.1) and (A.11).

Lemma A.2. In the same hypothesis of lemma A.1 the following estimate holds

$$
\sum_{i=1}^{2}\left\|v^{i}\right\|_{1, i}^{2} \leq C N^{2} \sum_{i=1}^{2}\left\|q^{i}\right\|_{0, i}^{2}
$$

for a positive constant $C$ independent of $q, \mathbf{v}$ and $N$.

Proof. Using (A.2) and (A.7) a direct computation gives, for $i=1,2$ :

$$
\begin{aligned}
\left\|q^{i}\right\|_{0, i}^{2} \geq & C\left(\left(x_{i}-x_{i-1}\right) \sum_{0 \leq k, l \leq N-1}\left(\alpha_{k l}^{i}\right)^{2} \frac{1}{(2 k+1)(2 l+1)}+\right. \\
& \left.+\frac{x_{i}-x_{i-1}}{N^{3}} \sum_{k=0}^{N-1}\left(\beta_{k}^{i}\right)^{2} \cdot \frac{1}{2 k+1}+\frac{\left(x_{i}-x_{i-1}\right)^{3}}{N^{3}} \sum_{l=1}^{N-1}\left(\gamma_{l}^{i}\right)^{2} \frac{1}{2 l+1}\right)
\end{aligned}
$$

where $C$ is a positive constant independent of $q, N$ and $i$. It is readily seen that

$$
\left\|\frac{\partial v_{1}^{i}}{\partial x}\right\|_{0, i}+\left\|\frac{\partial v_{2}^{i}}{\partial y}\right\|_{0, i} \leq C\left\|q^{i}\right\|_{0, i}, \quad i=1,2
$$

Let us now estimate $\left\|\frac{\partial v_{i}^{i}}{\partial y}\right\|_{0, i}$. From (A.1) and (A.11) we obtain

$$
\frac{\partial v_{1}^{i}}{\partial y}=\sum_{0 \leq l \leq k \leq N-1, k+l \neq 0} \alpha_{k l}^{i} J_{k}^{\prime} \otimes L_{l}^{\prime}+\sum_{k=1}^{N-1} \beta_{k}^{i} J_{k}^{i} \otimes L_{N-1}+B^{i} \otimes L_{N-1} .
$$

We now estimate the three terms appearing in the right hand side of (A.19). 
1) Recalling the inequality $\int_{-1}^{1}\left(L_{l}^{\prime}\right)^{2} d x \leq C(2 l+1)$, we get for $i=1,2$

$$
\begin{aligned}
& \left\|\sum_{0 \leq l \leq k \leq N-1} \alpha_{k l}^{i} J_{k}^{i} \otimes L_{l}^{\prime}\right\|_{0, i}^{2} \leq C\left[\sum_{l=0}^{N-1}(2 l+1)\left(\int_{x_{i-1}}^{x_{i}}\left(\sum_{k=l, k+l \neq 0}^{N} \alpha_{k l} J_{k}^{i}\right)^{2} d x\right)^{1 / 2}\right]^{2} \leq \\
& \leq C\left[\sum_{l=0}^{N-1}(2 l+1)\left(\sum_{k=l, k+l \neq 0}\left(\alpha_{k l}^{i}\right)^{2} \frac{\left(x_{i}-x_{i-1}\right)^{3}}{(2 k+1)^{3}}\right)^{1 / 2}\right]^{2} \leq \\
& \leq C\left[\sum_{l=0}^{N-1}(2 l+1)^{1 / 2}\left(\sum_{k=l}\left(\alpha_{k+l \neq 0}^{i}\right)^{2} \frac{\left(x_{i},-x_{i-1}\right)^{3}}{(2 k+1)(2 l+1)}\right)^{1 / 2}\right]^{2} .
\end{aligned}
$$

Using the Cauchy-Schwarz inequality and (A.17) we deduce

$$
\begin{aligned}
& \left\|\sum_{0 \leq l \leq k \leq N-1} \alpha_{k l}^{i} J_{k}^{i} \otimes L_{l}^{\prime}\right\|_{0, i}^{2} \leq \\
& \leq C\left(\sum_{l=0}^{N-1} 2 l+1\right)\left(\sum_{k=0}^{N-1}\left(\alpha_{k l}^{i}\right)^{2} \frac{\left(x_{i}-x_{x-1}\right)^{3}}{(2 k+1)(2 l-1)}\right) \leq C N^{2}\left\|q^{i}\right\|_{0, i}^{2}
\end{aligned}
$$

2) From (A.2) and (A.17) we obtain for $i=1,2$

$$
\left\|\sum_{k=1}^{N-1} \beta_{k}^{i} J_{k}^{i} \otimes L_{N-1}\right\|_{0, i}^{2} \leq \frac{C}{N} \sum_{k=1}^{N-1}\left(\beta_{k}^{i}\right)^{2} \frac{\left(x_{i}-x_{i-1}\right)^{3}}{(2 k+1)^{3}} C N^{2}\left\|q^{i}\right\|_{0, i}^{2} .
$$

3) Recalling (A.9) and using the Poincaré inequality, (A.10) and (A.17) we deduce

$$
\begin{aligned}
& \sum_{i=1}^{2}\left\|B^{i} \otimes L_{N-1}\right\|_{0, i}^{2} \leq \frac{C}{N} \int_{x_{0}}^{x_{2}} B^{2}(x) d x \leq \frac{C}{N} \sum_{i=1}^{2}\left(x_{i}-x_{i-1}\right)\left(\beta_{0}^{i}\right)^{2} . \leq \\
& \leq C N^{2} \sum_{i=1}^{2}\left\|q^{i}\right\|_{0, i}^{2} .
\end{aligned}
$$

From (A.19)-(A.22) we obtain

$$
\sum_{i=1}^{2}\left\|\frac{\partial v_{1}^{i}}{\partial y}\right\|_{0, i}^{2} \leq C N^{2} \sum_{i=1}^{2}\left\|q^{i}\right\|_{0, i}^{2}
$$

Using the same technique it is possible to estimate $\sum_{i=1}^{2}\left\|\frac{\partial v_{i}^{i}}{\partial x}\right\|_{0, i}^{2}$, hence, recalling (A.18) we obtain (A.16).

Lemma A.3. In the same hypothesis of lemma A.1 the following estimate holds

$$
\sum_{i=1}^{2}\left\|\mathbf{v}^{i} \cdot \tau^{i}\right\|_{L^{2}\left(\partial \Omega_{i}\right)^{3}} \leq C \sum_{i=1}^{2}\left\|q^{i}\right\|_{0, i}^{2}
$$


for a positive constant independent of $q, \mathbf{v}$ and $N$.

Proof. From (A.1) and (A.11) we obtain for $i=1,2$

$$
\forall x \in\left[x_{i-1}, x_{i}\right], \quad \mathrm{v}^{i} \cdot \tau_{4}^{i}(x, 1)=-\sum_{0 \leq l \leq k \leq N-1} \alpha_{k+l \neq 0}^{i} J_{k l}^{i}(x)+A^{i}(x) .
$$

Using (A.2), the Cauchy-Schwarz inequality and (A.17) we deduce

$$
\begin{aligned}
& \int_{x_{i-1}}^{x_{i}}\left(\sum_{0 \leq l \leq k \leq N-1, k+l \neq 0} \alpha_{k l}^{i} J_{k}^{i}(x)\right)^{2} d x \leq C \sum_{k=0}^{N-1}\left[\left(\sum_{l=0}^{k} \alpha_{k l}^{i}\right)^{2} \frac{\left(x_{i}-x_{i-1}\right)^{3}}{(2 k+1)^{3}}\right] \leq \\
& \leq C\left(x_{i}-x_{i-1}\right)^{3} \sum_{k=0}^{N-1}\left[\left(\sum_{l=0}^{k} \frac{\left|\alpha_{k l}^{i}\right|}{(2 k+1)^{1 / 2}(2 l+1)^{1 / 2}}\right)^{2} \frac{1}{2 k+1}\right] \leq \\
& \leq C\left(x_{i}-x_{i-1}\right)^{3} \sum_{k=0}^{N-1} \sum_{l=0}^{k} \frac{\left(\alpha_{k l}^{i}\right)^{2}}{(2 k+1)(2 l+1)} \frac{k+1}{2 k+1} \leq C\left\|q^{i}\right\|_{0, i}^{2} .
\end{aligned}
$$

The second term at the right hand side of (A.24) can be estimated using the technique of the proof of (A.22), so that we obtain

$$
\sum_{i=1}^{2}\left\|\mathrm{v}^{i} \cdot \tau_{4}^{i}\right\|_{L_{2}\left(\partial \Omega_{i}\right)} \leq C \sum_{i=1}^{2}\left\|q^{i}\right\|_{0, i}^{2} .
$$

Working in the same lines to estimate $\sum_{i=1}^{2}\left\|\mathrm{v}^{i} \cdot \tau_{j}^{i}\right\|_{L^{3}\left(\partial \Omega_{i}\right)}, \quad j=1,2,3$. we obtain (A.24).

Theorem A.1. Let $q \in P_{N}\left(\bar{\Omega}_{1}\right) \times P_{N}\left(\bar{\Omega}_{2}\right)$ be orthogonal to $\bar{Z}_{N, 2}$. There exists $\mathrm{w} \in V_{N}$ such that

$$
\nabla \cdot \mathbf{w}^{i}=q^{i} \quad \text { for } i=1,2
$$

and

$$
\sum_{i=1}^{2}\left\|w^{i}\right\|_{i, 1}^{2} \leq C N^{2} \sum_{i=1}^{2}\left\|q^{i}\right\|_{0, i}^{2},
$$

for a positive constant $C$ independent of $\mathrm{w}, q$ and $N$.

Proof. Let $\theta_{N}^{+}$and $\theta_{N}^{-}$be two functions such that

$$
\begin{gathered}
\theta_{N}^{+}, \theta_{N}^{-} \in P_{N}([-1,1]) \\
\theta_{N}^{+}( \pm 1)=0, \quad \theta_{N}^{-}( \pm 1)=0 \\
\left(\theta_{N}^{+}\right)^{\prime}(-1)=0, \quad\left(\theta_{N}^{-}\right)^{\prime}(-1)=1 \\
\left(\theta_{N}^{+}\right)^{\prime}(1)=1, \quad \theta_{N}^{\prime}(1)=0
\end{gathered}
$$


and

$$
\left\|\theta_{N}^{+}\right\|_{L^{2}(-1,1)}+\left\|\theta_{N}^{-}\right\|_{L^{2}(-1,1)} \leq \frac{C}{N^{3}} .
$$

One can for instance choose $\theta_{N}^{+}(y)=-\frac{(1+y)^{2}(1-y) L_{N}^{\prime \prime \prime}(y)}{4 L_{N}^{\prime \prime \prime}(1)}$ and $\theta_{N}^{-}(y)=-\theta_{N}^{+}(-y)$. (it can be proved that it is not possible to find $\theta_{N}^{+}$and $\theta_{N}^{-}$verifying (A.27) and (A.28) with on the right hand side a power of $N$ greater than 3 ).

Let $q$ and $\mathrm{v}$ be given by (A.7) and (A.11) respectively. We define the functions $f$ and $g$ as follows:

$$
\begin{gathered}
f(y)=v^{1} \cdot \tau_{4}^{1}\left(b_{1}\right) \theta_{N}^{+}(y)-v^{1} \cdot \tau_{2}^{1}\left(a_{1}\right) \theta_{N}^{-}(y) \\
g(y)=-\frac{\partial v^{1} \cdot \tau_{1}^{1}}{\partial \tau_{l}^{1}}\left(b_{1}\right) \theta_{N}^{+}(y)-\frac{\partial v^{1} \cdot \tau_{2}^{1}}{\partial \tau_{2}^{1}}\left(a_{i}\right) \theta_{N}^{-}(y) \quad \text { for } y \in[-1,1] .
\end{gathered}
$$

For $i=1,2$ we now define the functions $\phi^{i}$ and $\psi^{i}$ on $\partial \Omega_{i}$ as follows:

$$
\psi_{i}=0 \text { on } \partial \Omega_{1} \cap \partial \Omega
$$

$$
\begin{array}{cc}
\forall y \in[-1,1] & \psi^{i}\left(x_{1}, y\right)=f(y) \\
\psi^{i}=v^{i} \cdot \tau^{i} & \text { on } \partial \Omega_{1} \cap \partial \Omega
\end{array}
$$

$\forall y \in[-1,1], \quad \psi^{1}\left(x_{1}, y\right)=g(y)$ and $\psi^{2}\left(x_{1}, y\right)=\mathbf{v}^{1} \cdot \tau_{3}^{1}+\mathbf{v}^{2} \cdot \tau_{1}^{2}-g(y)$.

We now show that there exists $\Phi \in P_{N}\left(\bar{\Omega}_{1}\right) \times P_{N}\left(\bar{\Omega}_{2}\right)$ such that

$$
\left\{\begin{array}{l}
\Psi^{i}=\phi^{i} \text { on } \partial \Omega_{i} \\
\frac{\partial \Phi^{i}}{\partial n^{i}}=\psi^{i} \text { on } \partial \Omega_{i}
\end{array}\right.
$$

and

$$
\sum_{i=1}^{2}\left\|\Phi^{i}\right\|_{2, i}^{2} \leq C \sum_{i=1}^{2}\left(N^{6}\left\|\phi^{i}\right\|_{L^{2}\left(\partial \Omega_{i}\right)}^{2}+N^{2}\left\|\psi^{i}\right\|_{L^{2}\left(\partial \Omega_{i}\right)}\right) .
$$

The existence of this function $\Phi$ is a consequence of a trace theorem due to Bernardi and Maday provided some compatility conditions hold (see [4, thm 3.1]). To simplify the notation let us fix the index $i$ and set $A_{1}=a_{i-1}, A_{2}=a_{i}, A_{3}=b_{i}, A_{4}=b_{i-1}$ (see fig. A.1). We denote by $\phi_{j}^{i}$ (resp. $\psi_{j}^{i}$ ) the restriction of $\phi^{i}\left(\right.$ resp. $\left.\psi^{i}\right)$ to $\left[A_{j-1}, A_{j}\right]$. The compatibility condition are the following

$$
\begin{aligned}
& \phi_{j}^{i}\left(A_{j}\right)=\phi_{j+1}^{i}\left(A_{j}\right), \quad 1 \leq j \leq 4 \\
& \frac{\partial \phi_{j}^{i}}{\partial \tau_{j}^{i}}\left(A_{j}\right)=\psi_{j+1}^{i}\left(A_{j}\right), \quad 1 \leq j \leq 4 \\
& \frac{\partial \phi_{j+1}^{i}}{\partial \tau_{j}^{i}}\left(A_{j}\right)=-\psi_{j}^{i}\left(A_{j}\right), \quad 1 \leq j \leq 4 \\
& \frac{\partial \psi_{j}^{i}}{\partial \tau_{j}^{i}}\left(A_{j}\right)=-\frac{\partial \psi_{j+1}^{i}}{\partial \tau_{j+1}^{i}}\left(A_{j}\right), \quad 1 \leq j \leq 4 .
\end{aligned}
$$


Thanks to (A.27) (A.29) and (A.30) the conditions (A.33) are satisfied.

For $j=1$ the condition (A.34) reads as follows:

$$
\begin{gathered}
\mathbf{v}^{1} \cdot \tau_{2}^{1}\left(a_{0}\right)=0 \\
\mathbf{v}^{2} \cdot \tau_{2}^{2}\left(a_{1}\right)=-f^{\prime}(-1) .
\end{gathered}
$$

Due to (A.27) and (A.29) these relations can be written in the form

$$
\begin{gathered}
\mathbf{v}^{1} \cdot \mathrm{n}_{1}^{1}\left(a_{0}\right)=0 \\
\mathbf{v}^{2} \cdot \mathbf{n}_{1}^{2}\left(a_{1}\right)=-\mathbf{v}_{1} \cdot \mathbf{n}_{3}^{1}\left(a_{1}\right)
\end{gathered}
$$

hence they follow from (A.13) and (A.14). Using (A.13) (A.27) and (A.29) and working in the same lines the conditions (A.34) for $j=1, \ldots, 4$ and (A.35) can be easily verified. For $j=1$ the compatibility condition (A.36) reads as follows:

$$
\begin{gathered}
\frac{\partial \mathbf{v}^{1} \cdot \tau_{1}^{1}}{\partial \tau_{1}^{1}}\left(a_{0}\right)=-\frac{\partial \mathrm{v}^{1} \cdot \tau_{2}^{1}}{\partial \tau_{2}^{1}}\left(a_{0}\right) \\
\frac{\partial \mathbf{v}^{1} \cdot \tau_{3}^{1}}{\partial \tau_{1}^{2}}\left(a_{1}\right)+\frac{\partial \mathbf{v}^{2} \cdot \tau_{1}^{2}}{\partial \tau_{1}^{2}}\left(a_{1}\right)+g^{\prime}(-1)=-\frac{\partial \mathbf{v}^{2} \cdot \tau_{1}^{2}}{\partial \tau_{2}^{2}}\left(a_{1}\right) .
\end{gathered}
$$

The first condition can be written in the form $\nabla \cdot v^{1}\left(a_{0}\right)=0$. It follows from (A.12) and the orthogonality of $q^{1}$ to $\chi_{a_{0}}^{1}$. Recalling (A.29) and (A.30) the second condition is $\nabla \cdot v^{1}\left(a_{1}\right)=\nabla \cdot v^{2}\left(a_{1}\right)$. This is a consequence of (A.12) and the orthogonality of $q$ to $\left(\chi_{a_{1}}^{1},-\chi_{a_{1}}^{2}\right)$. Working in the same lines the relations (A.36) for $j=2,3,4$ can be proved. They follow from (A.12) and the orthogonality of $q$ to the elements $\left(0, \chi_{a_{2}}^{2}\right),\left(\chi_{b_{0}}^{1}, 0\right),\left(0, \chi_{b_{2}}^{2}\right)$ and $\left(\chi_{b_{1}}^{1},-\chi_{b_{1}}^{2}\right)$.

It follows from (A.33)-(A.36) that there exists $\Phi \in P_{N}\left(\bar{\Omega}_{1}\right) \times P_{N}\left(\bar{\Omega}_{2}\right)$ such that (A.31) and (A.32) hold. We set

$$
\mathbf{w}=\mathbf{v}+\operatorname{rot} \Phi,
$$

where $\mathrm{v}$ is given by (A.11). From lemma A.1 and (A.30) (A.31) we have that $\mathrm{w} \in V_{N}$ and $\nabla \cdot \mathrm{w}=q$. To prove (A.26) note that

$$
\sum_{i=1}^{2}\left\|w^{i}\right\|_{1, i}^{2} \leq C \sum_{i=1}^{2}\left(\left\|v^{i}\right\|_{1, i}^{2}+\|\Phi\|_{2, i}^{2}\right) .
$$

Recalling (A.32) we have to estimate the terms $\sum_{i=1}^{2}\left\|\phi^{i}\right\|_{L^{2}\left(\partial \Omega_{i}\right)^{2}}$ and $\sum_{i=1}^{2}\left\|\psi^{i}\right\|_{L^{3}\left(\partial \Omega_{i}\right)^{2}}$. Thanks to (A.28) (A.29) and (A.30) we have

$$
\sum_{i=1}^{2}\left\|\psi^{i}\right\|_{L^{2}\left(\partial \Omega_{i}\right)} \leq \frac{C}{N^{6}}\left(\left|\left(\mathbf{v}^{1} \cdot \tau_{4}^{i}\right)\left(b_{1}\right)\right|^{2}+\left|\left(\mathbf{v}^{1} \cdot \tau_{2}^{1}\right)\left(a_{1}\right)\right|^{2}\right)
$$


and

$$
\sum_{i=1}^{2}\left\|\psi^{i}\right\|_{L^{2}\left(\partial \Omega_{i}\right)} \leq \frac{C}{N^{6}}\left(\left|\frac{\partial \mathrm{v}^{1} \cdot \tau_{4}^{1}}{\partial \tau_{4}^{1}}\left(b_{1}\right)\right|^{2}+\left|\frac{\partial \mathrm{v}^{1} \cdot \tau_{2}^{1}}{\partial \tau_{2}^{1}}\left(a_{1}\right)\right|^{2}\right)+\sum_{i=1}^{2}\left\|v^{i} \cdot \tau^{i}\right\|_{L^{2}\left(\partial \Omega_{i}\right)}
$$

From (A.39) (A.1) (A.10) (A.11) and (A.17) we get

$$
\sum_{i=1}^{2}\left\|\phi^{i}\right\|_{L^{2}\left(\partial \Omega_{i}\right)} \leq \frac{C}{N^{6}}\left|A^{1}\left(x_{1}\right)\right|^{2} \leq \frac{C}{N^{6}}\left|\alpha_{00}^{1}\right|^{2} \leq \frac{C}{N^{6}}\left\|q^{1}\right\|_{0,1}^{2}
$$

From (A.1) (A.11) and (A.40) we obtain

$$
\sum_{i=1}^{2}\left\|\psi^{i}\right\|_{L^{2}\left(\partial \Omega_{i}\right)} \leq \frac{C}{N^{6}}\left(\sum_{0 \leq l \leq k \leq N-1} \mid \alpha_{k l}^{1} \|\right)^{2}+\sum_{i=1}^{2}\left\|v^{i} \cdot \tau^{i}\right\|_{L^{2}\left(\partial \Omega_{i}\right)}
$$

Using the Cauchy-Schwarz inequality, (A.17) and lemma A.3 we deduce

$$
\begin{aligned}
& \sum_{i=1}^{2}\left\|\psi^{i}\right\|_{L^{2}\left(a \Omega_{i}\right)} \leq \frac{C^{\prime}}{N^{2}} \sum_{0 \leq l \leq k \leq N-1}\left|\alpha_{k l}^{1}\right|^{2} \frac{1}{(2 k+1)(2 l+1)}+ \\
& +C^{\prime \prime} \sum_{i=1}^{2}\left\|q^{i}\right\|_{0, i}^{2} \leq C \sum_{i=1}^{2}\left\|q^{i}\right\|_{0, i}^{2} \cdot
\end{aligned}
$$

Finally from (A.37), (A.16), (A.38), (A.32), (A.41) and (A.42) we obtain (A.26).

We are now in a position to generalize the result of theorem A.1 to the case of $M$ aligned subdomains. To this end let us denote by $\tilde{Z}_{N, M}$ the subspace of $\prod_{i=1}^{M} P_{N}\left(\bar{\Omega}_{i}\right)$ spanned by the $4 M+4$ spurious modes given in (1.6)-(1.10). The other notation are those of section one.

Theorem A.R. Let $q \in \prod_{i=1}^{M} P_{N}\left(\widetilde{\Omega}_{i}\right)$ be orthogonal, for the inner product $((\cdot, \cdot))_{N}$ defined in (0.4), to any element of $\tilde{Z}_{N, M}$. Then there exists $w \in V_{N}$ such that

$$
\nabla \cdot w^{i}=q^{i} \quad \text { for } i=1, \ldots, M
$$

and

$$
\sum_{i=1}^{M}\left\|w^{i}\right\|_{1, i}^{2} \leq C N^{2} \sum_{i=1}^{M}\left\|q^{i}\right\|_{0, i}^{2}
$$

where $C$ is a positive constant independent of $\mathrm{w}, q$ and $N$.

Proof. We use an induction argument on $M$. Theorem A.1 gives the case $M=2$. We suppose now that the theorem holds for $M-1(M \leq 3)$ aligned subdomains and 
we consider the case of figure 1.1. The function $q \in \prod_{i=1}^{M} P_{N}\left(\bar{\Omega}_{i}\right)$ can be decomposed as follows:

$$
q=\bar{q}+\overline{\bar{q}}
$$

where

$$
\bar{q}^{1}=q^{1}, \quad \bar{q}^{i}=0 \quad \text { for } i=3, \ldots, M
$$

and $\bar{q}^{2}$ has the following properties

$$
\begin{gathered}
\bar{q}^{2}\left(a_{1}\right)=q^{1}\left(a_{1}\right), \quad \bar{q}^{2}\left(b_{1}\right)=q^{1}\left(b_{1}\right), \quad \bar{q}^{2}\left(a_{2}\right)=\bar{q}^{2}\left(b_{2}\right)=0, \\
\left(q^{1}, L_{0}^{1} L_{0}\right)_{N, 1}+\left(\bar{q}^{2}, L_{0}^{2} L_{0}\right)_{N, 2}=0, \quad\left(q^{1}, L_{N}(y)\right)_{N, 1}+\left(\bar{q}^{2}, L_{N}(y)\right)_{N, 2}=0, \\
\left(\bar{q}^{2}, L_{N}^{2}(x)\right)_{N, 2}=\left(\bar{q}^{2}, L_{N}^{2}(x) L_{N}(y)\right)_{N, 2}=0,
\end{gathered}
$$

there exists a positive constant $C$ independent of $N$ such that

$$
\left\|\bar{q}^{2}\right\|_{0,2} \leq C\left\|q^{1}\right\|_{0,1}
$$

The polynomial $\vec{q}^{2}$ is then defined by $\bar{q}^{2}=q^{2}-\bar{q}^{2}$. Let us assume for a moment that such a decomposition exists. From (A.45)-(A.48) it follows that $\left(q^{1}, \bar{q}^{2}\right)$ is orthogonal to $\tilde{Z}_{N, 2}$ and $\left(\bar{q}^{2}, q^{3}, \ldots, q^{M}\right)$ verifies the hypothesis of the theorem in the $M-1$ subdomains $\Omega_{2}, \ldots, \Omega_{M}$. Hence from theorem A.1 and the induction hypothesis there exists $\overline{\mathrm{w}} \in \prod_{i=1}^{2} P_{N}\left(\bar{\Omega}_{i}\right)^{2}$ and $\overline{\overline{\mathrm{w}}} \in \prod_{i=2}^{M} P_{N}\left(\bar{\Omega}_{i}\right)^{2}$ with $\overline{\mathrm{w}}=0$ on $\partial\left(\Omega_{1} \cup \Omega_{2}\right), \overline{\overline{\mathrm{w}}}=0$ on $\partial\left(\cup_{i=2}^{M} \Omega_{i}\right), \bar{w}$ and $\overline{\bar{w}}$ continuous at the interfaces, such that

$$
\nabla \cdot \overline{\mathrm{w}}^{i}=\vec{q}^{i}, \quad i=1,2, \quad \nabla \cdot \overline{\overline{\mathrm{w}}}^{i}=\bar{q}^{i}, \quad i=2, \ldots, M
$$

and

$$
\sum_{i=1}^{2}\left\|\mathrm{w}^{i}\right\|_{1, i}^{2} \leq C N^{2} \sum_{i=1}^{2}\left\|\vec{q}^{i}\right\|_{0, i}^{2} \quad, \quad \sum_{i=2}^{M}\|\overline{\mathrm{w}}\|_{1, i}^{2} \leq C N^{2} \sum_{i=2}^{M}\left\|\overline{\vec{q}}^{i}\right\|_{0, i}^{2} .
$$

We now set

$$
\mathrm{w}=\left\{\begin{array}{l}
\overline{\mathrm{w}}^{1} \text { in } \bar{\Omega}_{1} \\
\overline{\mathrm{w}}^{2}+\overline{\mathrm{w}}^{2} \text { in } \bar{\Omega}_{2} \\
\overline{\overline{\mathrm{w}}}^{i} \text { in } \bar{\Omega}_{i} \text { for } i=3, \ldots, M .
\end{array}\right.
$$

Clearly $\mathbf{w} \in V_{N}$, furthmore from (A.45) and (A.50) we have that $\nabla \cdot \mathbf{w}^{i}=q^{i}$ for $i=$ $1, \ldots, M$.

From (A.52) (A.51) and (A.46) we obtain

$$
\begin{aligned}
\sum_{i=1}^{M}\left\|\mathrm{w}^{i}\right\|_{1, i}^{2} & \leq C\left(\sum_{i=1}^{M}\left\|\overline{\mathrm{w}}^{i}\right\|_{1, i}^{2}+\sum_{i=2}^{M}\left\|\overline{\mathrm{w}}^{i}\right\|_{1, i}^{2}\right) \leq \\
& \leq C N^{2}\left(\sum_{i=1}^{M}\left\|q^{i}\right\|_{0, i}^{2}+\left\|\bar{q}^{2}\right\|_{0,2}^{2}+\left\|\overline{\bar{q}}^{2}\right\|_{0,2}^{2}\right)
\end{aligned}
$$


hence using (A.45) and (A.49) we get

$$
\sum_{i=1}^{M}\left\|\mathrm{w}^{i}\right\|_{1, i}^{2} \leq C N^{2}\left(\sum_{i=1, i \neq 2}^{M}\left\|q^{i}\right\|_{0, i}^{2}+\left\|q^{1}\right\|_{0,1}^{2}+\left\|q^{2}\right\|_{0,2}^{2}\right)
$$

which gives (A.44). To conclude the proof we exhibit a polynomial $\bar{q}^{2}$ which verifies (A.47) (A.48) and (A.49). We suppose, for simplicity, $N$ even and we set:

$$
\begin{aligned}
\bar{q}^{2}= & \left(\omega_{N}^{2}\right)^{2} \chi_{b_{1}}^{2} q^{1}\left(b^{1}\right)+\left(\omega_{N}^{2}\right)^{2} \chi_{a_{1}}^{2} q^{1}\left(a_{1}\right)+\alpha_{1} L_{0}^{2} \otimes L_{0}+\alpha_{2} L_{0}^{2} \otimes L_{N}+ \\
& +\alpha_{3} L_{N}^{2} \otimes L_{0}+\alpha_{4} L_{N}^{2} \otimes L_{N}+\alpha_{5} L_{2}^{2} \otimes L_{2}
\end{aligned}
$$

where $\alpha_{k}, k=1, \ldots, 5$, are constants.

From (0.7) it follows that (A.47) is verified provided $\alpha_{5}=-\sum_{k=1}^{4} \alpha_{k}$.

An easy computation shows that $\alpha_{k}, k=1, \ldots, 4$, can be found so that (A.48) holds, moreover we have

$$
\left|\alpha_{k}\right| \leq\left(\left\|q^{1}\right\|_{0,1}+\left(\omega_{N}^{2}\right)^{2}\left|q^{1}\left(b_{1}\right)\right|\left\|\chi_{b_{1}}^{2}\right\|_{0,2}+\left(\omega_{N}^{2}\right)^{2}\left|q^{1}\left(a_{1}\right)\right|\left\|\chi_{a_{1}}^{2}\right\|_{0,2}\right) .
$$

Hence from (A.53) we deduce

$$
\left\|\bar{q}^{2}\right\|_{0,2} \leq C\left(\left\|q^{1}\right\|_{0,1}+\left(\omega_{N}^{2}\right)^{2}\left(\left|q^{1}\left(b_{1}\right)\right|\left\|\chi_{b_{1}}^{2}\right\|_{0,2}+\left|q^{1}\left(a_{1}\right)\right|\left\|\chi_{a_{1}}^{2}\right\|_{0,2}\right)\right) .
$$

Using (0.2) and (0.3) it is readly seen that the two last terms can be bounded by $\left\|q^{1}\right\|_{0,1}$ hence (A.49) holds.

\section{Proof of Proposition 1.1 and Theorem 1.2.}

From theorem A.2 and (0.5) we have that there exists a positive constant $C$ independent of $N$ such that

$$
\forall q \in \tilde{Z}_{N, M}^{\perp} \quad \exists \mathrm{w} \in V_{N}: \frac{((q, \nabla \cdot \mathrm{w}))_{N}}{\|q\|_{N}\|\nabla \mathbf{w}\|_{N}} \geq C \frac{\|q\|_{0}}{\|\nabla \mathbf{w}\|_{0}} \geq \frac{C}{N}
$$

hence

$$
\inf _{q \in \tilde{Z}_{N, N}} \sup _{\mathbf{v} \in V_{N}} \frac{((q, \nabla \cdot \mathbf{v}))_{N}}{\|q\|_{N}\|\nabla \mathbf{v}\|_{N}} \geq \frac{C}{N}
$$

From (A.54) we deduce that $\tilde{Z}_{\bar{N}, M}^{\perp}$ do not contain any spurious mode for the pressure. Recalling (1.5) we then have $\tilde{Z}_{N, M}^{\perp} \cap Z_{N, M}=\{0\}$. This implies that $\operatorname{dim} Z_{N, M} \leq \operatorname{dim} \tilde{Z}_{N, M}$. Since $Z_{N, M} \supset \tilde{Z}_{N, M}$ we deduce that $Z_{N, M}=\tilde{Z}_{N, M}$, hence proposition 1.1 is proved and theorem 1.2 follows from (A.54) and (1.11). 
Remark A.1 (More complex decompositions).

The proof technique of theorem A.2 can be generalized to cover more complex decompositions. Consider for instance the L-shaped domain of figure A.2.a.

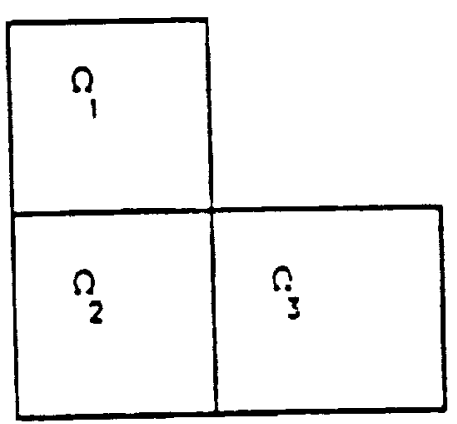

figure A.2.a

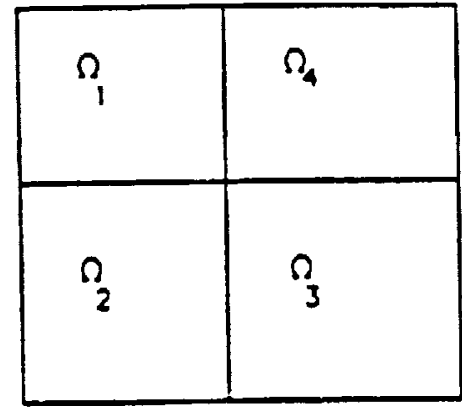

figure A.2.b

If $q \in M_{N}$ (the pressure space described in section 3 ) then it can be written in the form (A.45) with $\bar{q}^{1}=q^{1}, \overline{\bar{q}}^{3}=q^{3}$ and $\bar{q}^{2}$ such that $\left(\bar{q}^{1}, \bar{q}^{2}\right)$ belongs to $\tilde{Z}_{N, 2}^{\perp}$. It turns out that theorem A.1 can be applied in $\Omega_{1} \cup \Omega_{2}$ and in $\Omega_{2} \cup \Omega_{3}$ and that theorem A.2 holds for this decomposition. Analogous consideration holds for the figure A.2.b. In this case $q \in M_{N}$ can be written in the form $q=\bar{q}+\overline{\bar{q}}$ with $\bar{q}^{1}=0, \vec{q}^{4}=q^{4}, \bar{q}^{1}=q^{1}, \bar{q}^{4}=0$ and $\vec{q}^{i}, \bar{q}^{i}$ for $i=2,3$ such that one can apply theorem A.2 on the L-shaped domains $\Omega_{1} \cup \Omega_{2} \cup \Omega_{3}$ and $\Omega_{2} \cup \Omega_{3} \cup \Omega_{4}$. For a general partition of the domain of the kind of that of figure 4.1 one can always find a decomposition of $q$ as in the previous cases. Hence proposition 1.1 and theorem 1.2 can be proved also in this case. 


\section{References.}

[1] Adams R.A., Sobolev Spaces, Academic Press, New York, 1975.

[2] Brezzi $F$., On the existence, uniqueness and approximation of a saddle point problem arising from Lagrange multipliers. RAIRO Anal. Num., 8-R2, pp.129-151, 1974.

[3] Bernardi C., Canuto C., Maday Y., Generalized inf-sup conditions for Chebyschev approximations of the Navier-Stokes equations. To appear in SIAM J. Numer. Anal. 1988.

[4] Bernardi C., Maday Y., Relévement pôlynomial de traces et applications. Submitted to $M^{2} A N, 1988$.

[5] Bernardi C., Maday Y., Nonconforming Spectral Element methods, analysis of some projection operators. To appear in J. Applied Numer. Math., 1989.

[6] Bernardi C., Maday Y., Métivet B., Calcul de la pression dans la résolution spectrale du système de Stokes. La Recherche Aérospatiale, 1, pp.1-24, 1986.

[7] Canuto C., Funaro D., The Schwarz algorithm for spectral methods. SIAM J. Numer. Anal. 25, n.1, pp.24-40, 1988.

[8] Canuto C., Hussaini M.Y., Quarteroni A., Zang T.A., Spectral Methods in Fluid Dynamics. Springer-Verlag, New-York, 1987.

[9] Canuto C., Pietra P., Boundary and interface conditions within a Finite Element preconditioner for the Spectral Collocation method. To appear in J. Comp. Phys. 1988.

[10] Davis P.J., Rabinowitz P., Methods of Numerical Integration, Academic Press, New York, 1975.

[11] Funaro D., A multidomain spectral approximation of elliptic problems. Numer. Math. for PDE, 2, pp.187-205, 1986.

[12] Funaro D., Quarteroni A., Zanolli P., An iterative procedure with interface relaxation for domain decomposition methods. To appear in SIAM J. Numer. Anal. 1988.

[13] Gottlieb D., Orszag S.A., Numerical Analysis of Spectral Methods. SIAM, Philadelphia, 1977.

[14] Glowinski R., Wheeler M.F., In Domain Decomposition Methods for Partial Differential Equations, Glowinski R., Golub G.H., Periaux J., Eds. SIAM, Philadelphia, 1987.

[15] Métivet B., Résolution Spectrale des Equations de Navier-Stokes par une Méthode de Sousdomaines Courbes. Thèse, Univ. Paris VI, 1987.

[16] Maday Y., Patera A.T., Spectral Element methods for the incompressible NavierStokes equations. In State of the Art Surveys in Computational Mechanics, Noor A. Ed., ASME 1987.

[17] Marini D., Quarteroni A., A relaxation procedure for domain decomposition using Finite Elements. Submitted to Numer. Math. 1988. 
[18] Quarteroni, A., Sacchi Landriani G., Domain decomposition preconditioners for the spectral collocation method. J. Scient. Comp. Vol. 3, n. 1, 1989.

[19] Quarteroni A., Sacchi Landriani G., Parallel algorithm for the capacitance matrix method in domain decomposition. To appear in Calcolo 1989.

[20] Vandeven H., On the weakly spurious modes in a collocation method for the Stokes problem. To appear in_Mathematical Modelling and Numerical Analysis.

[21] Temam R., Navier Stokes Equations, North-Holland, 1977. 


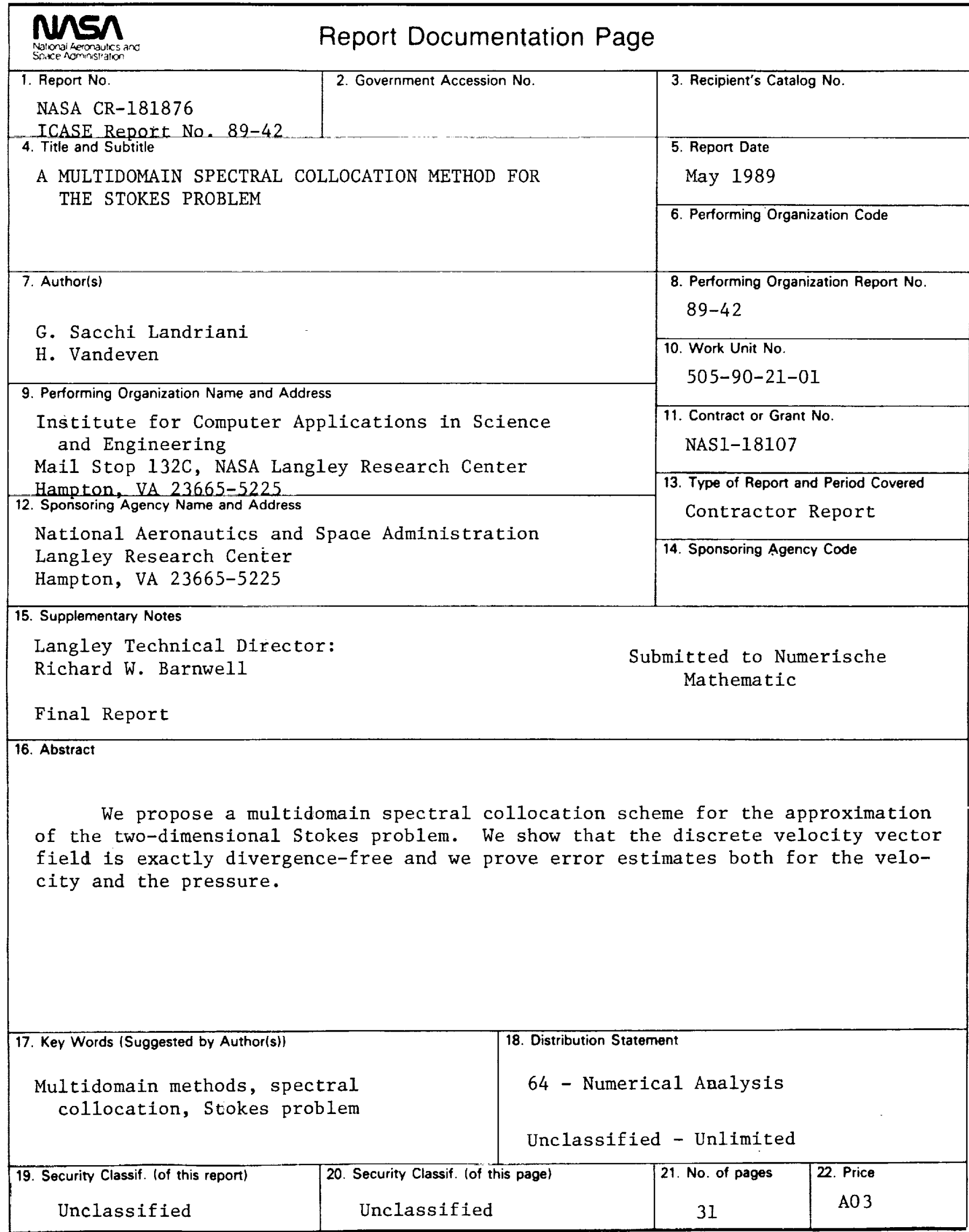

